\title{
Space density distribution of galaxies in the absolute magnitude - rotation velocity plane: a volume-complete Tully-Fisher relation from CALIFA stellar kinematics ${ }^{\star}$
}

\author{
S. Bekeraité ${ }^{1}$, C. J. Walcher ${ }^{1}$, J. Falcón-Barroso ${ }^{2,3}$, B. Garcia Lorenzo ${ }^{2}$, M. Lyubenova ${ }^{4,9}$, S. F. Sánchez ${ }^{6,7,5}$, \\ K. Spekkens ${ }^{8}$, G. van de Ven ${ }^{4}$, L. Wisotzki ${ }^{1}$, B. Ziegler ${ }^{10}$, J. A. L. Aguerri ${ }^{2,3}$, J. Barrera-Ballesteros ${ }^{2,3}$, \\ J. Bland-Hawthorn ${ }^{11}$, C. Catalán-Torrecilla ${ }^{12}$, R. García-Benito ${ }^{6}$, and the CALIFA collaboration \\ ${ }^{1}$ Leibniz-Institut für Astrophysik Potsdam (AIP), An der Sternwarte 16, 14482 Potsdam, Germany \\ e-mail: sbekeraite@aip.de \\ 2 Dept. Astrofísica, Universidad de La Laguna, C/ Astrofísico Francisco Sánchez, 38205 La Laguna, Tenerife, Spain \\ 3 Instituto de Astrofísica de Canarias, C/Vía Láctea s/n, 38200 La Laguna, Tenerife, Spain \\ ${ }^{4}$ Max-Planck Institute for Astronomy, Königstuhl 17, 69117 Heidelberg, Germany \\ 5 Instituto de Astronomía, Universidad Nacional Autonóma de Mexico, A.P. 70-264, 04510 México, D.F., Mexico \\ 6 Instituto de Astrofísica de Andalucía (CSIC), Glorieta de la Astronomía, s/n, 18008 Granada, Spain \\ 7 Centro Astronómico Hispano Alemán, Calar Alto, (CSIC-MPG), C/Jesús Durbán Remón 2-2, 04004 Almería, Spain \\ 8 Department of Physics, Royal Military College of Canada, PO Box 17000, Station Forces, Kingston, ON, K7K 7B4, Canada \\ 9 Kapteyn Astronomical Institute, Rijksuniversiteit Groningen, Postbus 800, 9700 AV Groningen, The Netherlands \\ ${ }^{10}$ University of Vienna, Türkenschanzstr. 17, 1180 Vienna, Austria \\ 11 Institute of Astronomy, School of Physics, University of Sydney, NSW 2006, Australia \\ 12 Departamento de Astrofísica y CC. de la Atmósfera, Universidad Complutense de Madrid, 28040 Madrid, Spain \\ Received 19 September 2015 / Accepted 18 May 2016
}

\begin{abstract}
We measured the distribution in absolute magnitude - circular velocity space for a well-defined sample of 199 rotating galaxies of the Calar Alto Legacy Integral Field Area Survey (CALIFA) using their stellar kinematics. Our aim in this analysis is to avoid subjective selection criteria and to take volume and large-scale structure factors into account. Using stellar velocity fields instead of gas emission line kinematics allows including rapidly rotating early-type galaxies. Our initial sample contains 277 galaxies with available stellar velocity fields and growth curve $r$-band photometry. After rejecting 51 velocity fields that could not be modelled because of the low number of bins, foreground contamination, or significant interaction, we performed Markov chain Monte Carlo modelling of the velocity fields, from which we obtained the rotation curve and kinematic parameters and their realistic uncertainties. We performed an extinction correction and calculated the circular velocity $v_{\text {circ }}$ accounting for the pressure support of a given galaxy. The resulting galaxy distribution on the $M_{r}-v_{\text {circ }}$ plane was then modelled as a mixture of two distinct populations, allowing robust and reproducible rejection of outliers, a significant fraction of which are slow rotators. The selection effects are understood well enough that we were able to correct for the incompleteness of the sample. The 199 galaxies were weighted by volume and large-scale structure factors, which enabled us to fit a volume-corrected Tully-Fisher relation (TFR). More importantly, we also provide the volume-corrected distribution of galaxies in the $M_{r}-v_{\text {circ }}$ plane, which can be compared with cosmological simulations. The joint distribution of the luminosity and circular velocity space densities, representative over the range of $-20>M_{r}>-22$ mag, can place more stringent constraints on the galaxy formation and evolution scenarios than linear TFR fit parameters or the luminosity function alone.
\end{abstract}

Key words. galaxies: kinematics and dynamics - galaxies: statistics - galaxies: evolution

\section{Introduction}

The Tully-Fisher relation (TFR, Tully \& Fisher 1977) links two intrinsic properties of rotationally supported galaxies: their circular rotation velocities, and their luminosities. Stated in physical terms, this relation indicates a close relation between the total dynamical mass and the stellar mass (or the total baryonic content, McGaugh et al. 2000) of the galaxies.

Circular velocities and luminosities of galaxies have long been used to estimate extragalactic distances (see Opik 1922, for the first use), also see Roberts (1969), Bottinelli (1971),

\footnotetext{
* Galaxies main parameters and FITS files of 2D distributions are only available at the CDS via anonymous ftp to

cdsarc.u-strasbg.fr (130.79.128.5) or via

http://cdsarc.u-strasbg.fr/viz-bin/qcat?J/A+A/593/A114
}

Balkowski et al. (1974) and Shostak (1975) for early analyses of scaling relations of spiral galaxies.

The low intrinsic scatter of the TFR cannot be explained by initial conditions (Eisenstein \& Loeb 1996), which implies that the subsequent evolutionary processes were crucial in determining the shape of the relation. The fact that the TFR exists is thought to be a natural outcome of hierarchical structure assembly (Steinmetz \& Navarro 1999).

Although the TFR has primarily been envisaged and successfully used as a tool to determine extragalactic distances (Tully \& Fisher 1977), it also offers fundamental insights into the processes of disk assembly and evolution. We summarize the many uses of TFR in the following paragraphs.

The TFR in its initial form and in its many variants (relations between different measures of rotational velocity and the stellar 
mass, total baryonic mass, absolute magnitude in different passbands) has been extensively employed as a constraint on galaxy formation and evolution models (Koda et al. 2000; Croton et al. 2006; Dutton \& van den Bosch 2009; Dutton et al. 2011; Tonini et al. 2011; McCarthy et al. 2012; Vogelsberger et al. 2013). It also provided independent constraints on cosmological parameters (Eisenstein \& Loeb 1996; van den Bosch 2000; Masters et al. 2006), has been used to test the predictions of $\Lambda$ CDM (Blanton et al. 2008), and to characterise the properties of dark matter haloes such as their concentration (Dutton et al. 2011) and response to galaxy formation (Dutton \& van den Bosch 2009; Chan et al. 2015). The TFR has also been used to place constraints on virial properties of barred and unbarred galaxies (Courteau et al. 2003), on disk submaximality (Courteau \& Rix 1999; Courteau \& Dutton 2015), to investigate the origin of S0 galaxies (Neistein et al. 1999; Williams et al. 2009; Tonini et al. 2011), test the universality of the initial mass function (Bell $\&$ de Jong 2001; Dutton et al. 2011), and to infer the galaxy velocity function (Gonzalez et al. 2000).

In addition, TFR measurements at higher redshifts provided insights into the mode of gas accretion at $z \approx 2.2$ (Cresci et al. 2009), the stellar-to-dynamical mass ratio at $z \approx 3$ (Gnerucci et al. 2011), disk assembly timescales (Miller et al. 2012), the evolution of bulgeless galaxies (Miller et al. 2013), the "downsizing" effect (Böhm \& Ziegler 2007), and the luminosity evolution of rotating disks (Ziegler et al. 2002; Puech et al. 2008; Miller et al. 2011).

Reproducing the observed redshift evolution, slope, offset, and intrinsic scatter of the TFRs is a standard test of cosmological simulations. It has been a long-standing problem of cosmological simulations (Steinmetz \& Navarro 1999; van den Bosch 2000; Koda et al. 2000; Cole et al. 2000; Eke et al. 2001; Croton et al. 2006; Courteau et al. 2007; Dutton et al. 2011), but this problem has been significantly remedied by a combination of more sophisticated feedback implementations, prescriptions for dark halo response, and the increased accuracy of cosmological parameters.

Several studies have described the convergence on reproducing the observed TFR: Dutton et al. (2007), Governato et al. (2007), Trujillo-Gomez et al. (2011), Tonini et al. (2011), McCarthy et al. (2012), Vogelsberger et al. (2014). Semianalytical models by Tonini et al. (2011) reproduced the TFR at higher redshifts, but yield too bright values at $z=0$, probably as a result of the uncertainties in star formation histories. Hydrodynamical zoom-in re-simulations by McCarthy et al. (2012) reproduced the TFR for galaxies with $\log \left(M_{*}\right)<10.7$. The authors claimed that the turn-off at the higher mass end is due to the lack of AGN feedback prescription. Governato et al. (2007) employed $N$-body SPH simulations with supernova feedback to produce disk galaxies that lie on both the $I$ band and the baryonic TFR. Trujillo-Gomez et al. (2011) used Bolshoi dark-matter-only simulations and abundance matching to demonstrate that the luminosity-velocity relation and the baryonic TFR match the observed ones. The Illustris project implements a sophisticated feedback model that includes both stellar and AGN feedback, reporting slightly too high circular velocities in the $M_{*}-v_{\text {circ }}$ relation (Vogelsberger et al. 2014). The goal of this study is to provide a measurement of the TFR and $M_{r}-v_{\text {circ }}$ distribution that is best suited for a comparison with these theoretical predictions.

Given the large body of literature on the TFR, we need to justify revisiting the relation. The Calar Alto Legacy Integral Field Area (CALIFA) provides three main reasons, two related to the observational data type at hand (integral field spectroscopy - IFS), and the last one tied to the available sample properties.

First, optical long-slit observations have traditionally been the observational basis for the TFR analysis. Long-slit observations have the drawback of not being able to view the entire velocity field of a galaxy and thus are prone to being affected by non-circular velocity field distortions. IFS data allow using the full velocity information available to correct for nonaxisymmetric velocity field features, characterise the specific angular momentum of galaxies, and distinguish between disturbed velocity fields, pristine disks, and slow rotators. The possibility of performing the sample selection using kinematic properties of galaxies is more relevant to the TFR than visual morphological classification. This has strikingly been confirmed by Flores et al. (2006). These authors showed that the very large scatter in the intermediate-redshift TFR, previously reported from long- or multi-slit spectroscopic observations, is a result of modelling a large portion $(\approx 65 \%)$ of galaxies with anomalous kinematics. A similar point has been made by Andersen \& Bershady (2003), who demonstrated that galaxies with large kinematic and photometric asymmetries in their velocity fields tend to be offset from the TFR. Using 2D velocity fields also prevents slit misalignment with the semi-major axis of the galaxy, even if such differences are not expected be a major problem in the TFR context (Amram et al. 1994; Courteau 1997; Giovanelli et al. 1997; Barrera-Ballesteros et al. 2014).

In addition, the observed line-of-sight rotation velocities must be de-projected to obtain the true circular rotation velocities. However, inclination is a notoriously difficult parameter to measure and is frequently the largest source of uncertainty in circular velocity measurement (Schommer et al. 1993; Garrido et al. 2004; Giovanelli et al. 1997; Obreschkow \& Meyer 2013). In most of the Tully-Fisher studies, inclination estimates are obtained from the variously defined apparent axis ratios $b / a$ in the following way (Hubble 1926):

$\cos (i)^{2}=\frac{\left(\frac{b}{a}\right)^{2}-q^{2}}{1-q^{2}}$.

Here $q$ is the intrinsic axis ratio of the galaxy, which is different for different galaxy morphological types (e.g. Rodríguez \& Padilla 2013), but a mean value of $q=0.2$ is frequently used (Tully \& Pierce 2000). However, there are several shortcomings of this method. It frequently overestimates the inclination for face-on galaxies because any irregularity (such as the spiral arms, bars, and disk asymmetries) at the outskirts of a galaxy will make the $b / a$ seem higher (e.g. Maller et al. 2009). For galaxies that are observed close to edge-on, photometric inclination estimates suffer from uncertainty in the intrinsic axis ratio $q$, which depends on the Hubble type of the galaxy and evolves through cosmic time (Obreschkow \& Meyer 2013). However, this is not thought to be very significant in the context of TFR (Courteau 1997; Hall et al. 2012).

We are able to circumvent these difficulties with assumptions made for photometric inclination estimates and directly model the velocity fields as rotating disks, obtaining kinematic inclination and position angles as free parameters. These methods were first used a few decades ago by radio (Rogstad et al. 1976; Bosma 1978) and Fabry-Perot interferometry observers (Marcelin \& Athanassoula 1982; Bland et al. 1987; Nicholson et al. 1992; Schommer et al. 1993; Amram et al. 1994).

To our knowledge, the first analysis of TFR using 2D IFS velocity fields was reported by Courteau et al. (2003) for of barred and unbarred disk galaxies. The authors used the SparsePak IFU 
(Bershady et al. 2004) to test whether the rotation velocities measured using long-slit spectroscopy are reliable. Andersen \& Bershady (2003) derived a face-on Tully-Fisher relation based on $24 \mathrm{H} \alpha$ velocity fields of low-inclination $\left(16^{\circ}-41^{\circ}\right)$ galaxies. They showed that kinematic inclination estimates are sufficiently accurate down to $\approx 15^{\circ}$ and that this approach allows avoiding systematic and random errors arising from use of photometric axis ratio-based inclination estimates. For example, Schommer et al. (1993) found that photometric inclination estimates are systematically larger than inclinations derived from kinematics for galaxies with inclinations of up to $50^{\circ}$. As shown in this paper, by combining the photometric and kinematic data, we can model the velocity fields consistently and obtain the full distributions of parameter uncertainties for inclinations, position angles, and kinematic parameters, including the estimated rotation velocity.

Although several other IFS-based Tully-Fisher studies exist, they tend to focus on higher redshifts and have small sample sizes, aiming to investigate the dynamical state of high-redshift galaxies, assembly times of rotating disks, and morphological evolution (Swinbank et al. 2006; Puech et al. 2008; Cresci et al. 2009; Gnerucci et al. 2011). Similarly, Green et al. (2014) used the TFR measured from $\mathrm{H} \alpha$ velocity fields of local gasrich galaxies to gain insight into their high-redshift analogues. Recently, Di Teodoro et al. (2016) used a sample of $18 z 1$ KMOS galaxies to demonstrate that the TFR obtained from $\mathrm{H} \alpha$ emission is identical to the present one.

Therefore, the third major reason to revisit the TFR is that the CALIFA survey has better-defined sample selection criteria than earlier published work. In virtually all past TFR studies, the authors aimed for a clean sample, meaning a set of late (usually $\mathrm{Sa}$ or later) morphological type galaxies with ordered circular motions (e.g. Courteau 1996; Tully \& Pierce 2000; McGaugh et al. 2000). The goal of most of these studies was to estimate or calibrate a template Tully-Fisher relation that would be best suited for distance measurements. Many methods to account for selection effects in TFR samples have been developed, such as employing galaxy cluster observations to obtain volumecomplete samples, using the so-called inverse fitting methods, performing corrections based on morphology and extinction estimates, also attempting to account for varying distances to a cluster centre, implicit sample incompleteness, and many other possible sources of bias (see Giovanelli et al. 1997; Tully \& Pierce 2000; Verheijen 2001; Masters et al. 2006; Saintonge \& Spekkens 2011, and references therein for in-depth discussions of these methods and their shortcomings). While the above approaches are justified when the goal is to obtain a tight linear distribution with the least possible amount of scatter, our objective is different. We aim to use data-driven modelling to obtain a volume-corrected 2D distribution of rotationally supported galaxies. While such a distribution would not be directly useful for distance measurements (although we provide the parameters of a standard linear fit), it could place more stringent constraints on galaxy evolution models than the standard TFR or luminosity and velocity functions separately.

A sample selected for this purpose needs to span the widest possible range of morphologies (Verheijen 2001). The wish to include galaxies of all types and be able to perform consistent volume and large-scale structure corrections has compelled us to use the stellar velocity fields in this study. Stellar velocity fields have rarely been used for TFR measurements, mainly because of their lower signal-to-noise ratio $(\mathrm{S} / \mathrm{N})$ level. Emission lines are more easily detected in spectroscopic data, meaning that velocity fields based on emission line kinematics will extend farther out in the disk and have better spatial resolution. However, the main advantage of stellar velocity fields is that they can be obtained for galaxies without significant gas emission lines, that is, for early-type galaxies. A significant percentage of early type galaxies follows the TFR: for example, Krajnović et al. (2008) stated that about $80 \%$ of early-type galaxies and S0s have a rotatingdisk component. Similarly, Emsellem et al. (2011) showed that the majority of early type galaxies in the ATLAS3D sample are fast rotators, while Davis et al. (2011) demonstrated that earlytype galaxies lie on the CO Tully-Fisher relation, albeit likely offset from the one derived for spirals.

CALIFA is the first IFS survey to include many late-type galaxies as well, to have a large and statistically well-defined sample, and a sufficiently large field of view, all of which is necessary for this type of analysis.

\section{Data, sample selection, and characterisation}

\subsection{CALIFA survey}

CALIFA (Sánchez et al. 2012; Walcher et al. 2014, hereafter W14) is a legacy IFS survey of 600 nearby galaxies. Observations use the PMAS instrument (Roth et al. 2005) in PPaK (Verheijen et al. 2004) mode, mounted on the $3.5 \mathrm{~m}$ telescope at the Calar Alto observatory. The sample of galaxies that are observed with CALIFA is drawn from a larger pool of galaxies, selected from the SDSS DR7 survey, and termed the mother sample (MS). The MS is primarily diameter-limited and aimed at using the detector area efficiently. The CALIFA selection criteria are described in W14 in more detail. One salient feature of the CALIFA MS is that its selection criteria are well understood, providing us with a representative sample of galaxies that can be corrected for selection effects down to an absolute $r$-band magnitude of -19 mag. By adopting a probabilistic approach to outlier rejection, we show in this paper that we can keep a similar property of the sample even as we restrict it to conform to more stringent criteria.

The CALIFA data have been reduced using the CALIFA pipeline, and we refer to Sánchez et al. (2012), Husemann et al. (2013), García-Benito et al. (2015) for all the details. The result of the data reduction is two spectral cubes of the target galaxy, one in the V1200 grating and one in the V500 grating, which can be used to extract kinematic information. V1200 grating has been used here because this setup allowed measuring the velocity dispersion down to $\approx 50 \mathrm{~km} \mathrm{~s}^{-1}$.

Kinematic information of the stars was extracted from CALIFA datacubes using the pPXF fitting procedure (Cappellari $\&$ Emsellem 2004) and INDO-US (Valdes et al. 2004) spectral templates library. Bad pixels, foreground contamination, lowquality spaxels with $S / N<3$, emission line regions in the spectra and regions outside $3750-4650 \AA$ range (for the V1200 setup) were excluded from the fit. Spatial Voronoi binning (Cappellari $\&$ Copin 2003) was applied to ensure a constant $S / N=20$ in velocity dispersion, and it led to variable size bins with diameters ranging from $0.1 \mathrm{kpc}$ to $21 \mathrm{kpc}$ in linear size. On average, the galaxies have 131 useful kinematic data points, with the lowest number being 1 (galaxies with too few Voronoi bins were excluded from further analysis, as described in the next paragraphs) and the highest number of bins being 760 . The velocity and velocity dispersion values for each bin and the associated uncertainties were derived using $100 \mathrm{MCMC}$ realisations of the fit. We refer to the first paper of the CALIFA stellar kinematics series (Falcón-Barroso et al. 2016) where the kinematic map extraction is described in full detail. 

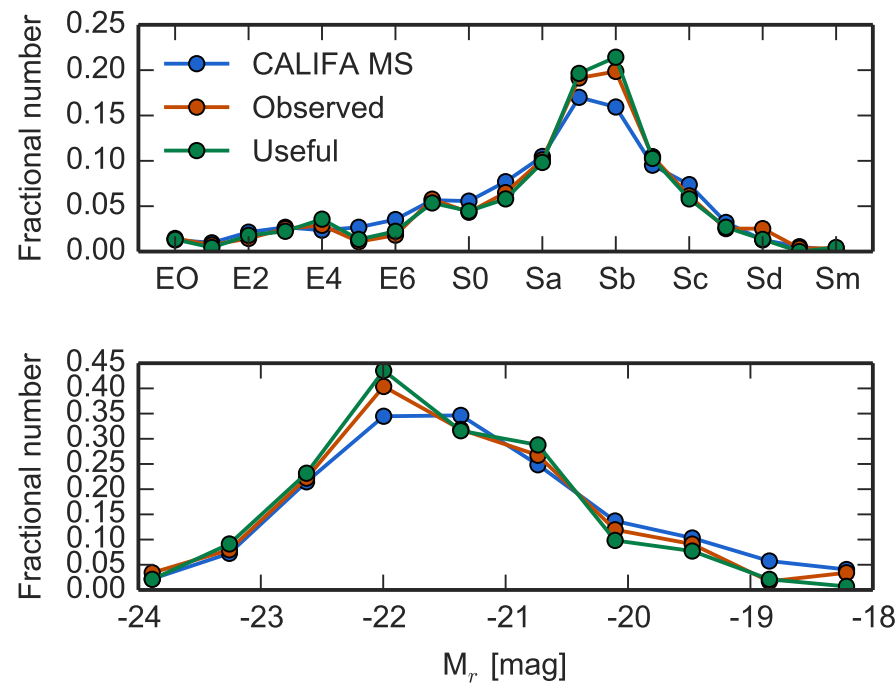

Fig. 1. Normalised histograms of SDSS Petrosian $M_{r}$ and morphological types of the CALIFA mother sample, observed sample (as of October 2013), and the useful subsample that we included in our analysis.

We started with the sample of galaxies observed by CALIFA until October 2013. This corresponded to 277 objects with derived stellar velocity fields. We term this the observed sample. Out of these, 51 were rejected at the beginning as not usable. A significant fraction of the rejected galaxies, 17, had too few bins $\left(N_{\text {bins }}<6\right)$ to even try to reliably constrain the rotation curves with the stellar velocity fields. In addition, we excluded 31 galaxies whose velocity fields could not provide a physical model. This included several heavily masked mergers, 4 galaxies where foreground objects obscured a significant part of the velocity map, 2 galaxies with significant dust lanes that obscured velocity fields, and the 5 galaxies that were excluded because they are unsuitable for the volume-correction procedure described below. We emphasize that the rejection here was not directly related to the internal properties of the galaxies that were relevant to this analysis (absolute magnitude, stellar mass, and rotation velocity), but to problematic observational data that precluded making realistic models. The resulting sample contains 226 useful velocity fields, and we refer to it as the useful sample in the following sections.

Figure 1 shows the normalised SDSS Petrosian absolute magnitude $M_{r}$ and morphological type histograms for the CALIFA mother sample, the observed sample, and the useful sample. As shown in the top panel, the observed sample contains slightly more $\mathrm{Sa}-\mathrm{Sb}$ type galaxies than the CALIFA mother sample. Even though the observing selection should be random, observing constraints and spatial variation within the sample volume might have introduced this discrepancy.

The lower panel of Fig. 1 shows normalised histograms of $M_{r}$. The observed and especially the useful sample lack the least luminous galaxies that are included in the mother sample. This effect in the useful sample is exacerbated because we were more likely to reject intrinsically fainter, later-type galaxies at this step, which were more likely to have fewer Voronoi bins. This must affect the outcome of the volume correction we perform at later steps.

\subsection{Volume corrections}

The CALIFA sample is limited by two main selection criteria, including all galaxies within the SDSS DR7 footprint that have (i) redshifts within $0.005<z<0.03$; and (ii) isophotal angular extents within $40^{\prime \prime}<\theta<79^{\prime \prime}$. . This construction principle allows us to perform volume corrections using the $V_{\max }$ method (Schmidt 1968) in much the same way as with a flux-limited sample (see W14 for details). While the sample as a whole is not volume complete, each galaxy can be assigned a well-defined accessible survey volume $V_{\max }$ over which it would be included given its properties and given the sample selection criteria.

It is important to realise that the selection by apparent diameter in CALIFA does by no means introduce a bias in terms of linear sizes of the galaxies in the sample because of the broad redshift range. Within the completeness range of the sample $\left(-19>M_{r}>-23.1\right.$; see W14), low-luminosity and small galaxies match the angular diameter criterion close to the low-redshift limit, while more luminous and larger galaxies occupy higher redshifts. By adding the contributions $1 / V_{\max }$ of all galaxies in suitable bins, we can calculate a volume-corrected estimate of a distribution function of the galaxy population. W14 demonstrated that the galaxy luminosity function as well as the size distribution function estimated in this way from the CALIFA mother sample are in excellent agreement with results from SDSS.

Another potential source of bias is related not to the sample selection process, but to the properties of the particular cosmological volume a given survey is probing. The CALIFA survey samples two nearby clusters (Virgo and Coma) as well as the underdensities in between, resulting in significant radial number density variations. W14 showed how these radial variations can be quantified and absorbed into effective volume correction factors. All volume-corrected quantities shown in this paper use these effective $V_{\max }$ values.

In this paper we also exploit the concept of volume corrections for the Tully-Fisher relation. Since our useful sample is much smaller than the CALIFA MS, the volume-correction factors need to be adjusted to reflect the size of the subsample. As long as the sample is a random subset of the mother sample, it is sufficient to reduce $V_{\max }$ by the sampling rate; this concept was used in the first two CALIFA data releases to verify that the released subsets (of 100 and 200 galaxies, respectively) are consistent in their statistical distribution properties with the mother sample and with the galaxy population as a whole (Husemann et al. 2013; García-Benito et al. 2015). In Fig. 2 we used the same approach to compare the galaxy luminosity function constructed from our useful sample with the results from the CALIFA MS and from SDSS. The agreement is excellent for absolute magnitudes $M_{r}<-20 \mathrm{mag}$, but there seem to be too few galaxies in the bins fainter than -20 mag for the useful sample. As mentioned above, this is probably due to our rejection of some late-type galaxies from the kinematic analysis because they have an insufficient number of Voronoi elements. Apart from this caveat, we conclude that our sample can be seen as volume-representative for the local galaxy population, at least for absolute magnitudes $-22<M_{r}<-20$.

Five more galaxies were excluded from further analysis: NGC 4676B and NGC 5947 had been added to the CALIFA MS by hand (see W14) and have no associated $V_{\max }$ values, while for NGC 7625, NGC 1056, and NGC 3057 these values are disproportionally low $\left(<10^{4} \mathrm{Mpc}^{3}\right)$. Since the $1 / V_{\max }$ weights are then correspondingly large, these few galaxies would totally dominate any $1 / V_{\max }$-weighted fit to the Tully-Fisher relation. We note that this is an inherent weakness of the $V_{\max }$ method. While more sophisticated approaches are conceivable that are more robust against such statistical fluctuations, we simply decided 


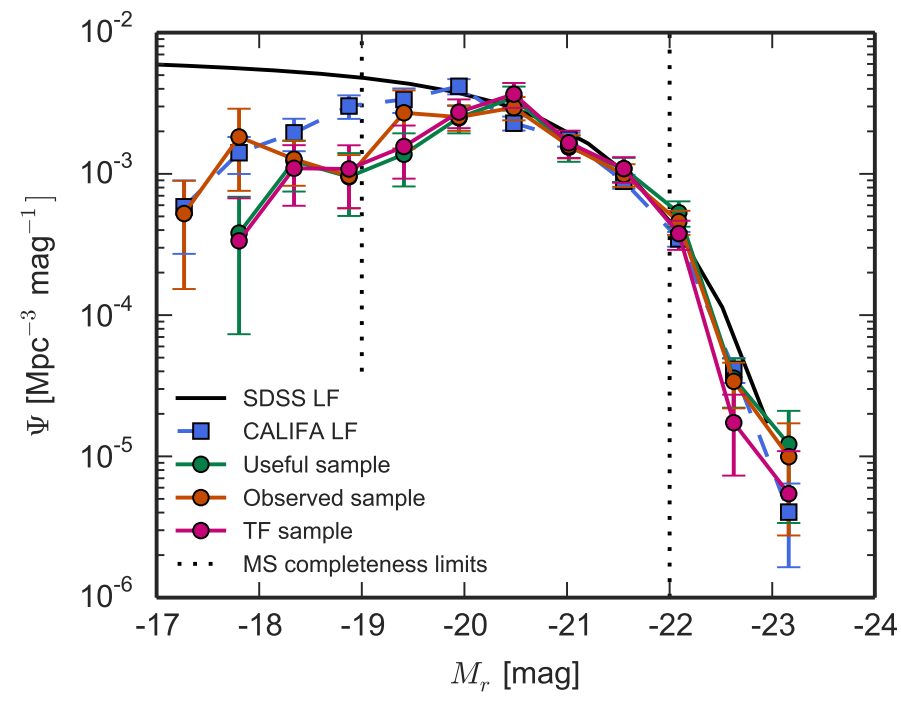

Fig. 2. Luminosity functions of the CALIFA mother sample (MS), the observed sample, the useful sample (see text), and the final Tully-Fisher sample defined in Sect. 5.2. The dotted lines denote the MS completeness limits calculated in W14. SDSS DR7 Petrosian $r$-band magnitudes were used for comparison with the SDSS luminosity function. The effects of outlier rejection steps are evident, especially for the more numerous low-luminosity galaxies with higher $1 / V_{\max }$ weights.

to remove these three objects. The effects of using or omitting $1 / V_{\max }$ weights when fitting the TFR are shown in Sect. 6.

\section{Luminosity data}

\subsection{Observed magnitudes}

We used the $r$-band growth curve photometry measurements described in W14. The uncertainties account for the combination of the contributions from the dark current and read noise, Poissonian sky counts error, uncertainties due to sky subtraction, and an estimate of uncertainties arising from masked foreground objects. The formal errors due to shot noise contribute only little to the error budget because of the large apparent sizes of our galaxies in the SDSS images. The magnitudes were corrected for Galactic extinction using SDSS pipeline values.

Absolute magnitudes were calculated using the prescriptions presented in W14. In short, the redshifts were corrected for Virgo-centric, Shapley and Great Attractor infall motions using the model by Mould et al. (2000). $K$-corrections were determined from spectral energy distributions as described in W14 and Walcher et al. (2008). The distance uncertainties were derived from group velocity dispersions obtained by the collaboration in W14 and combined with the photometric uncertainties when calculating the absolute magnitude uncertainties.

\subsection{Corrections for intrinsic absorption}

The absolute magnitudes need to be corrected for internal extinction, which depends on the inclination, bandpass, and morphology in a non-trivial way. We adopted the methods described in Wild et al. (2011a,b), which provide dust attenuation as a function of the photometric axis ratio $b / a$, the specific star formation rate, and the presence or absence of a significant bulge. We used the star formation rates determined from CALIFA $\mathrm{H} \alpha$ line emission based on the prescriptions of Calzetti (2013; Catalán-Torrecilla et al. 2015), photometric

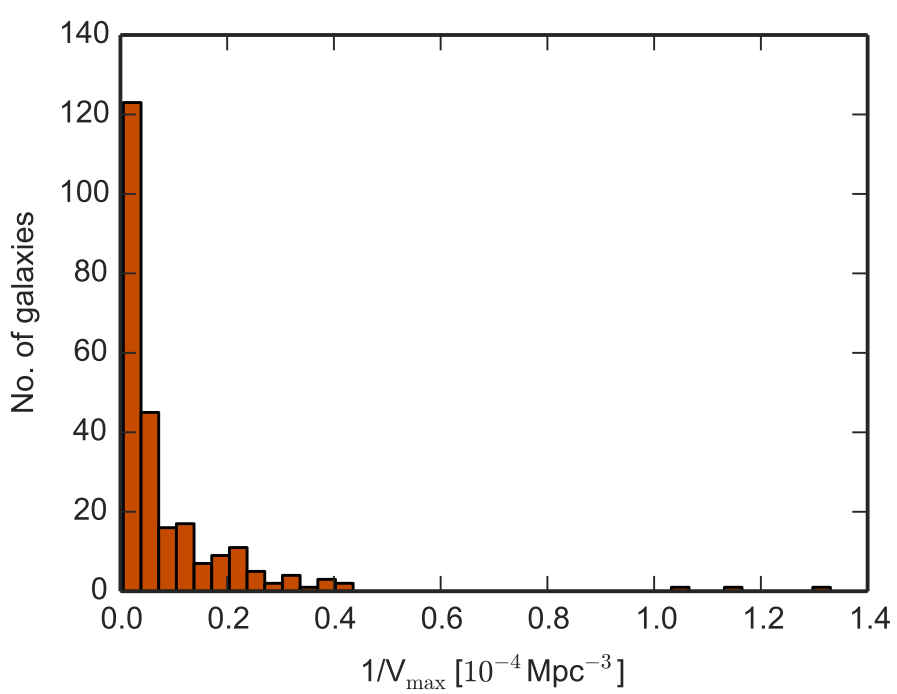

Fig. 3. Volume and radial-density weight factor $1 / V_{\max }$ histogram. The three outliers with $1 / V_{\max }>0.0001$ were excluded from further analysis.

stellar masses determined by the collaboration (W14) and integrated CALIFA $\mathrm{H} \alpha$ and $\mathrm{H} \beta$ fluxes. The corrected magnitude $M_{r}^{c}$ was calculated as $M_{r}^{c}=M_{r}+\Delta M_{r}$.

To correct the magnitudes for intrinsic attenuation, we needed the Balmer decrement of our sample galaxies. The emission line properties of $30^{\prime \prime}$ radius aperture spectra were extracted from the V500-datacubes of the galaxies. This aperture is large enough to include virtually $100 \%$ of the FoV of the CALIFA datacubes, without the need to select a different aperture for each galaxy.

To extract the information contained in the spectra, we followed the procedures described in Sánchez et al. (2014), using the fitting package FIT3D ${ }^{1}$.

Individual emission line fluxes were measured using FIT3D in the stellar-population-subtracted spectra performing a multicomponent fitting using a single Gaussian function. By subtracting a stellar continuum model derived with a set of SSP templates, we corrected for the effect of underlying stellar absorption, which is particularly important in Balmer lines (such as $\mathrm{H} \beta$ ).

Visual morphological classifications were used to distinguish between galaxies with and without significant bulges, which have different dust correction prescriptions. We assumed that galaxies that had been classified as Sc and later had no significant bulge. The correspondence between the visual morphological classification and the magnitude of the extinction correction is shown in Fig. 4.

It should be noted that we used kinematic inclination values obtained in the following section elsewhere in the analysis, especially when correcting the rotation curves for inclination. However, the methods described in Wild et al. (2011a,b) were derived using the photometric axis ratio as an input, therefore we employed it in our analysis for the sake of consistency.

We clipped the star formation rates and axis ratios to the maximum values provided in Wild et al. (2011a; $0.3<b / a<0.9$, $-10.2<\log (s S F R)<-9.3 \mathrm{yr}^{-1}$ for bulge-dominated galaxies and $-10.0<\log (s S F R)<-9.1 \mathrm{yr}^{-1}$ for disk-dominated galaxies $)$.

The CALIFA $\mathrm{H} \alpha$ and $\mathrm{H} \beta$ fluxes are not reliable at the low $\mathrm{S} / \mathrm{N}$ limit, leading to unrealistic Balmer decrements. We settled on making a $\mathrm{S} / \mathrm{N}$ cut at $\mathrm{H} \alpha / \mathrm{H} \beta=2.7$ ( $\mathrm{S} / \mathrm{N}$ was very close

1 http://www. caha.es/sanchez/FIT3D/ 


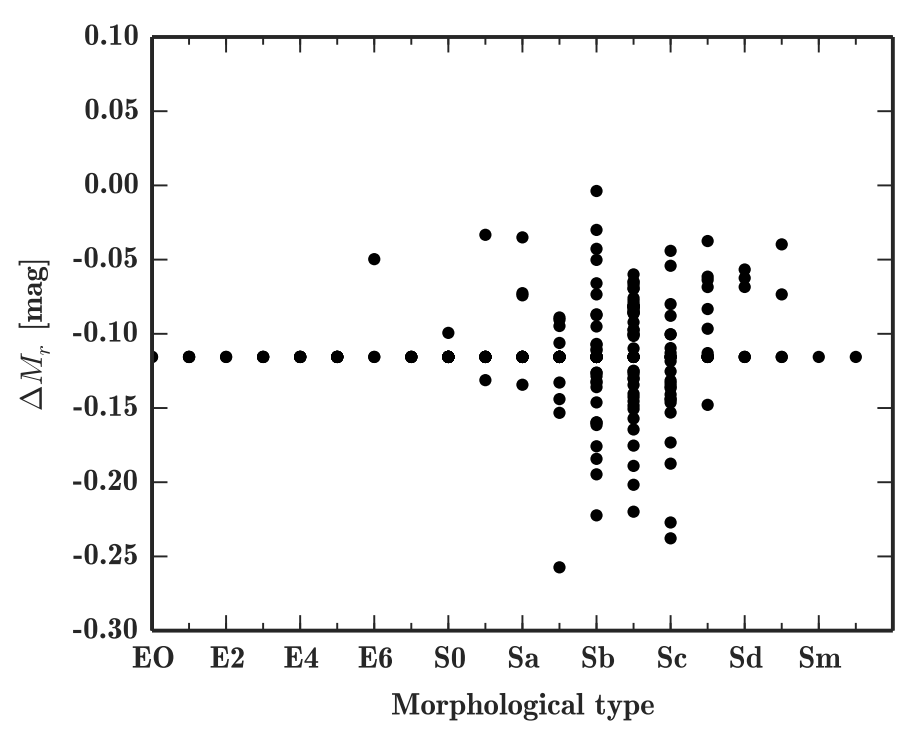

Fig. 4. Extinction correction vs. visual morphological classification.

to 4 there, see Fig. 5 for an illustration). For galaxies below the $S / N=4$ limit and for those that had no reliable $\mathrm{H} \alpha$ or $\mathrm{H} \beta$ fluxes, we adopted the average extinction correction value $\Delta M_{r}=-0.11 \mathrm{mag}$.

\section{Velocity field modelling}

\subsection{Model description}

It has long been noted that the deprojected rotation curves of galaxies show a variety of shapes (Rubin et al. 1985; Verheijen 2001), exhibiting differences attributed to morphology (Rubin et al. 1985; Verheijen 2001) or luminosity (Persic \& Salucci 1991). Several parameterisations of rotation curves exist, some being purely phenomenological (Courteau 1997; Vogt et al. 1996; Rix et al. 1997), some attempting physical parameterisation (Persic \& Salucci 1991; van den Bosch et al. 2000; Persic et al. 1996).

We used a variant of the arctan function (Courteau 1997), the hyperbolic tangent (Neumayer et al. 2011):

$v(r)=v_{0}+\frac{2}{\pi} v_{c} \cdot \tanh \left[\frac{r}{k \cdot r_{50}}\right]$,

where $v_{0}$ is the recession velocity, $v_{c}$ is a free parameter governing the amplitude of the rotation curve, $k$ describes the sharpness of RC turnover, and $r_{50}$ is the optical half-light semi-major axis, determined from $r$-band growth curve photometry. In addition, a galaxy was allowed to have arbitrary inclination and position angles. We did not allow the kinematic centre position to vary because the spatial resolution of Voronoi bins is variable and sometimes too low to provide a meaningful constraint on the centre position.

The main attraction of this model was its simplicity, that is, the lowest number of free parameters. However, we found that this simple model was unable to fit the rising or falling rotation curves.

At the cost of parameter degeneracy, we assumed another model for RC shapes, implemented in Bertola et al. (1991) and also discussed in Böhm et al. (2004):

$v(r)=v_{0}+\frac{v_{c} r}{\left(r^{2}+k^{2}\right)^{\frac{\gamma}{2}}}$.

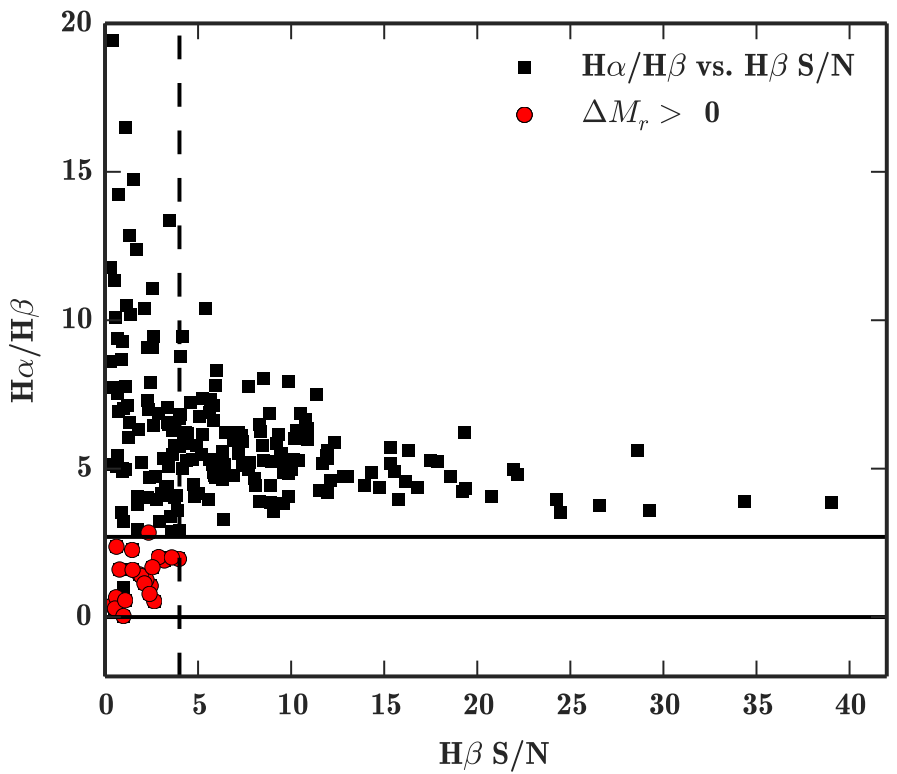

Fig. 5. Balmer decrement vs. $\mathrm{H}_{\beta} \mathrm{S} / \mathrm{N}$. The red points are the galaxies that had unreliable extinction corrections due to their low $\mathrm{S} / \mathrm{N}$ and correspondingly erroneous Balmer decrement values. The horizontal and vertical dashed lines show the region of probably untrustworthy extinction correction estimates. We used the mean magnitude of the extinction correction for these galaxies (see text).

The model has four free parameters $-v_{c}, k, v_{0}$, and $\gamma$. The $v_{c}$ and $k$ parameters here take on similar roles as in the tanh model (Eq. (2)), but their values are different. The $\gamma$ parameter, which typically varies between 0.8 and 1.2 , allows modelling rising and falling rotation curves. A flat rotation curve is obtained when $\gamma=1$.

In most optical studies the TFR defines the rotation velocity as measured at the outer part of a galaxy where the rotation curves are no longer rising. To achieve this, we had to extrapolate the rotation curves of galaxies that were not sampled up to the point of turnover.

We took advantage of an open-source Python implementation (Foreman-Mackey et al. 2013) of affine-invariant MCMC sampler (Goodman \& Weare 2010), called through a customised wrapper. MCMC methods provide the full posterior distributions of model parameters, leading to more realistic uncertainty estimates.

To constrain the physical parameters of the models, we applied a truncated Gaussian prior on the $\gamma$ parameter, effectively constraining it to lie between $0.8-1.2$. We found that this range of $\gamma$ values describes the range of physically possible rotation curves well and helps avoid degeneracies. In addition, we constrained the marginalised rotation velocity at 2.2 scale lengths $\left(v_{2.2}\right)$ to be below $600 \mathrm{~km} \mathrm{~s}^{-1}$, and restricted $k$ to $k<0$.

Even with the simplest hyperbolic tangent model, we observed a strong coupling between the inclination angle and the $v_{c}$ parameter, that is, the amplitude of the rotation curve. This is expected because almost any velocity field can potentially be modelled as a fast-rotating, almost face-on disk or as an inclined disk with a lower intrinsic rotation velocity if the spatial resolution is low. This proved to be a problem for highly inclined galaxies with a small number of bins (Fig. 6) because the inclination could not be constrained well.

To break this degeneracy, we introduced a truncated Gaussian prior on the inclination angle for galaxies with $i_{\text {phot }}>$ $75^{\circ}$. We estimated the prior inclination using Eq. (1) and the 

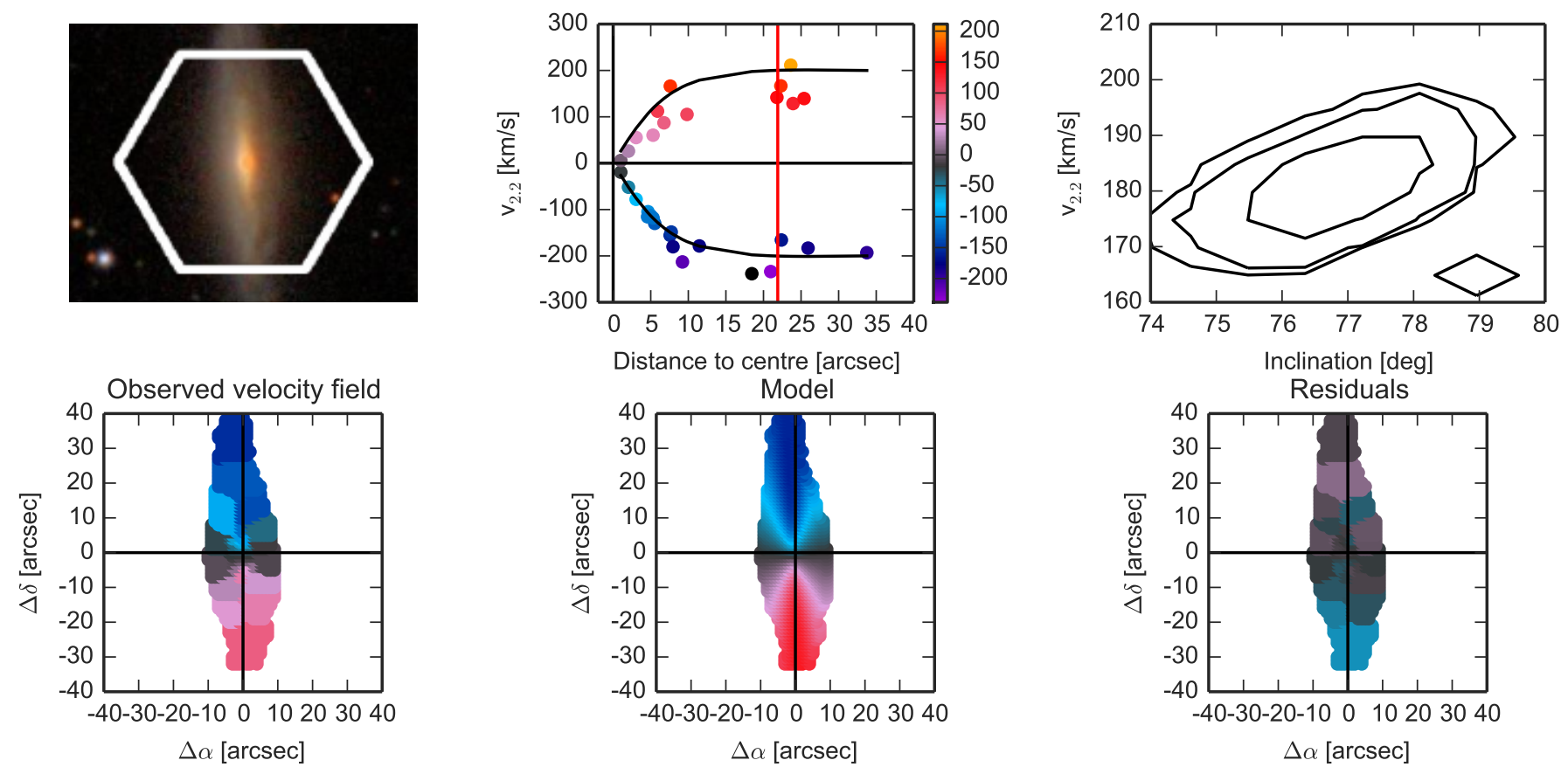

Fig. 6. Velocity field of an inclined galaxy (IC5376, $i_{\text {phot }}=80^{\circ}$ ). Top: SDSS composite image (left), model (black) and measured rotation curves (middle), joint $i-v_{2.2}$ distribution (right, shown as 1, 2, and 3 standard deviation contours). Bottom: observed velocity field (left), model (middle), and residuals (right). The red line indicates the location of $2.21_{\mathrm{sc}}$. The velocity scale is the same in all panels.

photometric axis ratios provided in $\mathrm{W} 14$, assuming the intrinsic disk thickness $q=0.2$ and the standard deviation of the Gaussian $\sigma=3^{\circ}$.

All photometric axis ratios were inspected visually and found to be quite accurate inclination indicators for highly inclined galaxies. This is not necessarily the case for lowinclination galaxies that can look less circular than they are as a result of spiral arms and other irregularities.

We noted a strong bimodality in the marginalised $v_{2.2}$ distribution for a number of objects. It occurred either as a consequence of a low number of Voronoi bins and therefore poorly constrained inclination, or of the inability to constrain the model based on kinematic information alone. In these cases, an identical truncated Gaussian prior was placed to constrain the inclination (and, consequently, $v_{2.2}$ ) to a more plausible range.

We took 480000 MCMC samples for each galaxy, rejecting the first 160000 to reduce the effect of the choice of initial parameters. The chain lengths were chosen after repeated modelling had shown that the models selected were robust, meaning that the parameter distributions did not change between fits. The MCMC outputs provide the full distributions of rotation curve parameters, inclination, and position angle values for each galaxy. From these we can obtain the marginalised posterior distribution of the modelled velocity at a given radius, as well as kinematic inclination and position angle estimates. Two examples of observed and model velocity fields, as well as their rotation curves and joint inclination- $v_{2.2}$ distributions, are shown in Figs. 6 and 7.

The mean values and standard deviations of kinematic inclination and position angles were determined through directional statistics, that is, by calculating the vector means and circular standard deviations of the chain values. Although the inclination and position angles were allowed to vary freely during fitting, the resulting chain values were wrapped to intervals $[0 ; 90]$ and $[0 ; 180]$, respectively.

\subsection{Definition of rotation velocity measure}

When the rotation velocity is measured at the outer parts of a galaxy, the TFR links the halo properties and the baryonic mass. However, in practice it is difficult to connect the true haloinduced velocity and the measured velocity because the radial coverage is limited (Verheijen 2001). The rotation velocity has to be measured at a particular point of the rotation curve, which affects the slope of the TFR (Yegorova \& Salucci 2007). It is often measured at $r_{2.2}=2.2 l_{\mathrm{sc}}$, where $l_{\mathrm{sc}}$ is the exponential scale radius of the disk, as well as at $r_{\mathrm{opt}}$, the radius containing $83 \%$ of all light (Courteau 1997). Other non-parametric definitions are also employed, for example, the maximum rotation velocity $v_{\max }$, rotation velocity at the flat part of the rotation curve $\left(v_{\text {flat }}\right)$, and the mean value of the outermost points of a rotation curve (see Böhm et al. 2004 for a discussion).

Two practical measures are $v_{2.2}$ and $v_{\text {opt }}$. We were reluctant to use $v_{\max }, v_{\text {flat }}$ and other non-parametric measures of the circular velocity because outlier points on the rotation curves were frequent as a result of Voronoi binning or lack of masking and not all the rotation curves were asymptotically flat. We decided to use $v_{\text {opt }}$ as our velocity measure because it is straightforward to compare with simulations and other observations as opposed to measuring $v_{2.2}$, which requires structural decomposition of a galaxy.

The mean coverage of CALIFA velocity fields, that is, the largest radius divided by $r_{\mathrm{opt}}$, is shown in Fig. 9 .

In addition, we checked whether the galaxies with lower spatial coverage are offset from the TFR. We did not find a significant offset (see Fig. 18 in Sect. 6).

\subsection{Modelling results and uncertainties}

For each galaxy we estimated the rotation velocity $v_{\text {opt }}$ by choosing between the two models described in Sect. 4.1. For the 

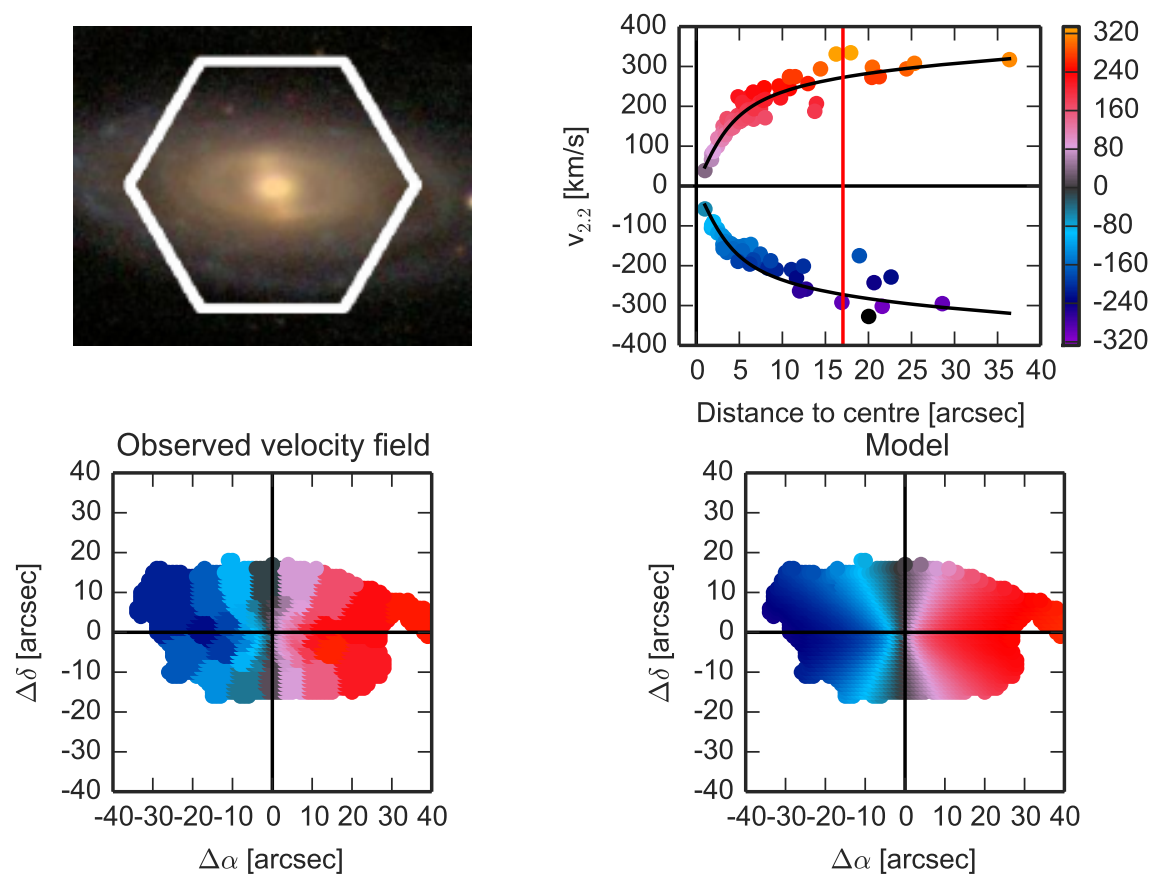
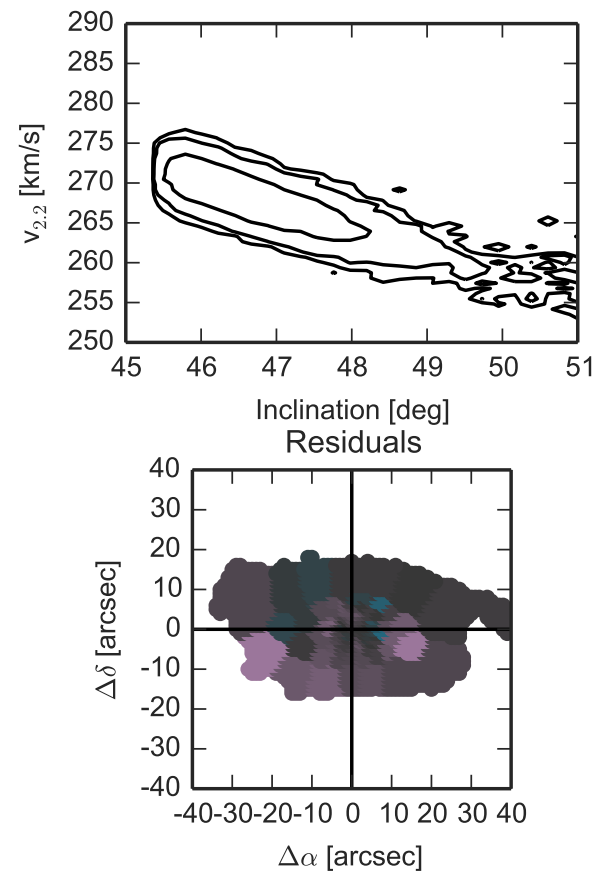

Fig. 7. Top: SDSS composite image of NGC 1645 (left), model (black), and measured rotation curves (middle), joint $i-v_{2.2}$ distribution (right). Bottom: observed velocity field (left), model (middle), and residuals (right). The photometric inclination was estimated to be equal to $52^{\circ}$.

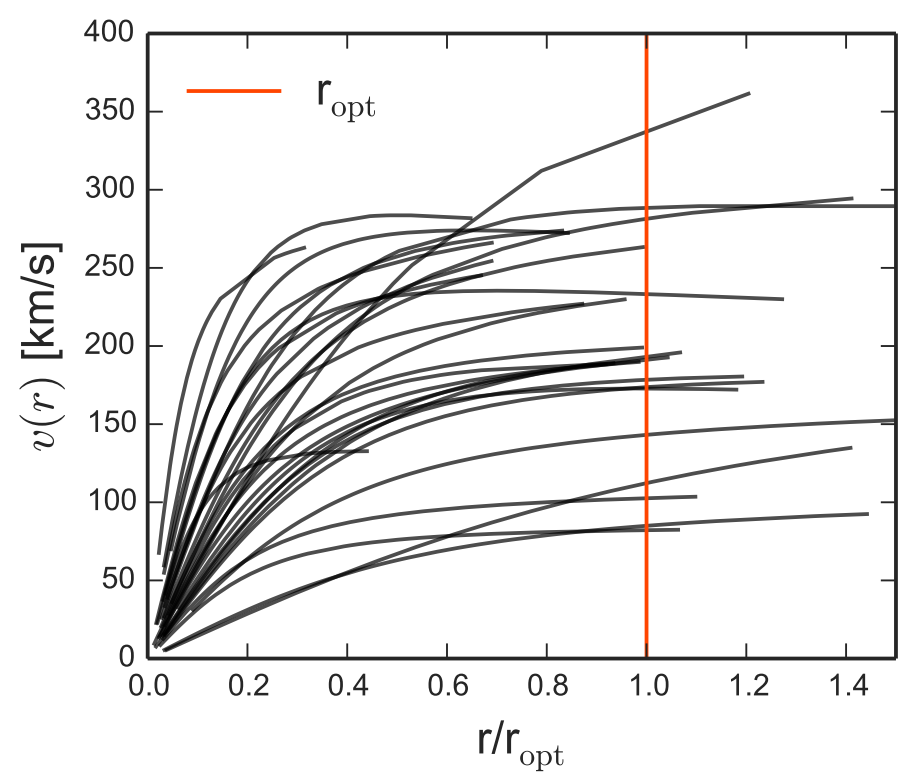

Fig. 8. Random sampling of 30 rotation curves scaled to $r_{\text {opt }}$, shown as the vertical line.

absolute majority (all except nine) of the galaxies, the more complex Bertola et al. (1991) model was preferable. In the three cases when both models were clearly incorrect in the outer parts of the galaxies, we picked the mean value of the last two points and added $20 \mathrm{~km} \mathrm{~s}^{-1}$ to the rotation velocity uncertainty.

Figure 10 shows the comparison of kinematic and photometric inclination estimates. As expected (Schommer et al. 1993), the photometric inclination estimates are systematically higher for low-inclination galaxies because any irregularity in the apparent light distribution forces the axis ratio towards lower values. At higher inclinations, inclination estimates of galaxies classified as mergers and slow rotators tended to differ the most.

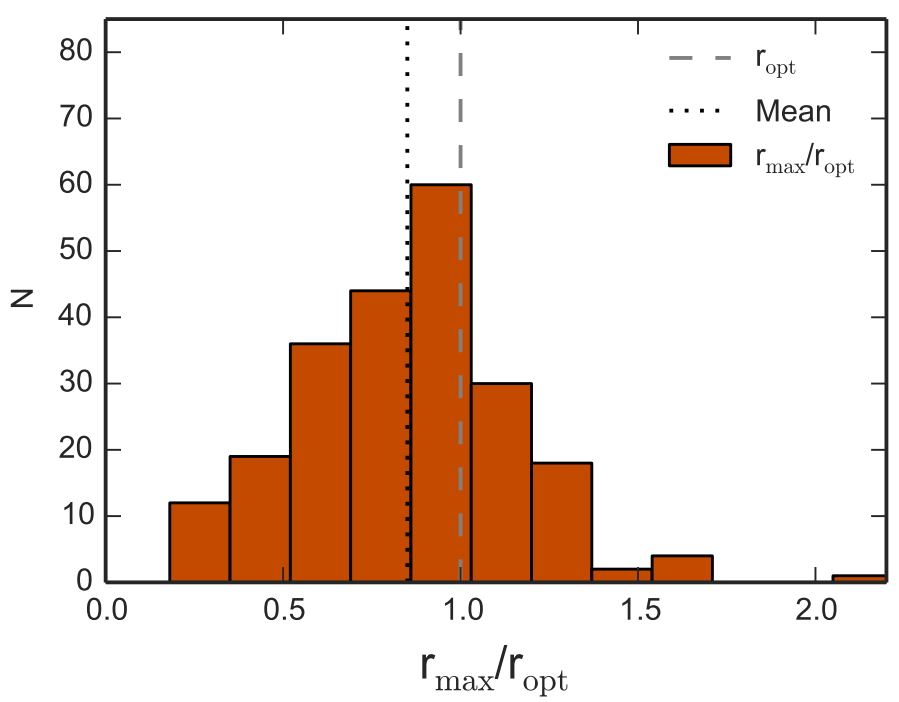

Fig. 9. Relative spatial coverage histogram of CALIFA stellar velocity fields. The dotted line denotes the mean value within the sample, the dashed line is at $r_{\mathrm{opt}}$.

Nevertheless, this does not present a problem for TF studies because the intrinsic rotation velocity is obtained by dividing the line-of-sight rotation velocity by the sine of the inclination angle, and the slope of the sine function is shallow for angles above $75^{\circ}$. We employed an identical Gaussian prior as above for the majority of galaxies with $i \geq 75^{\circ}$, therefore kinematic and photometric estimates tend to converge at the highest inclinations.

To estimate the reliability of $v_{\text {opt }}$ measurements, we discuss the potential sources of uncertainties. MCMC modelling provides estimates for uncertainties inherent in modelling a particular galaxy.

For some galaxies the main source of velocity uncertainties was the limitations of the simple rotating disk model. For 

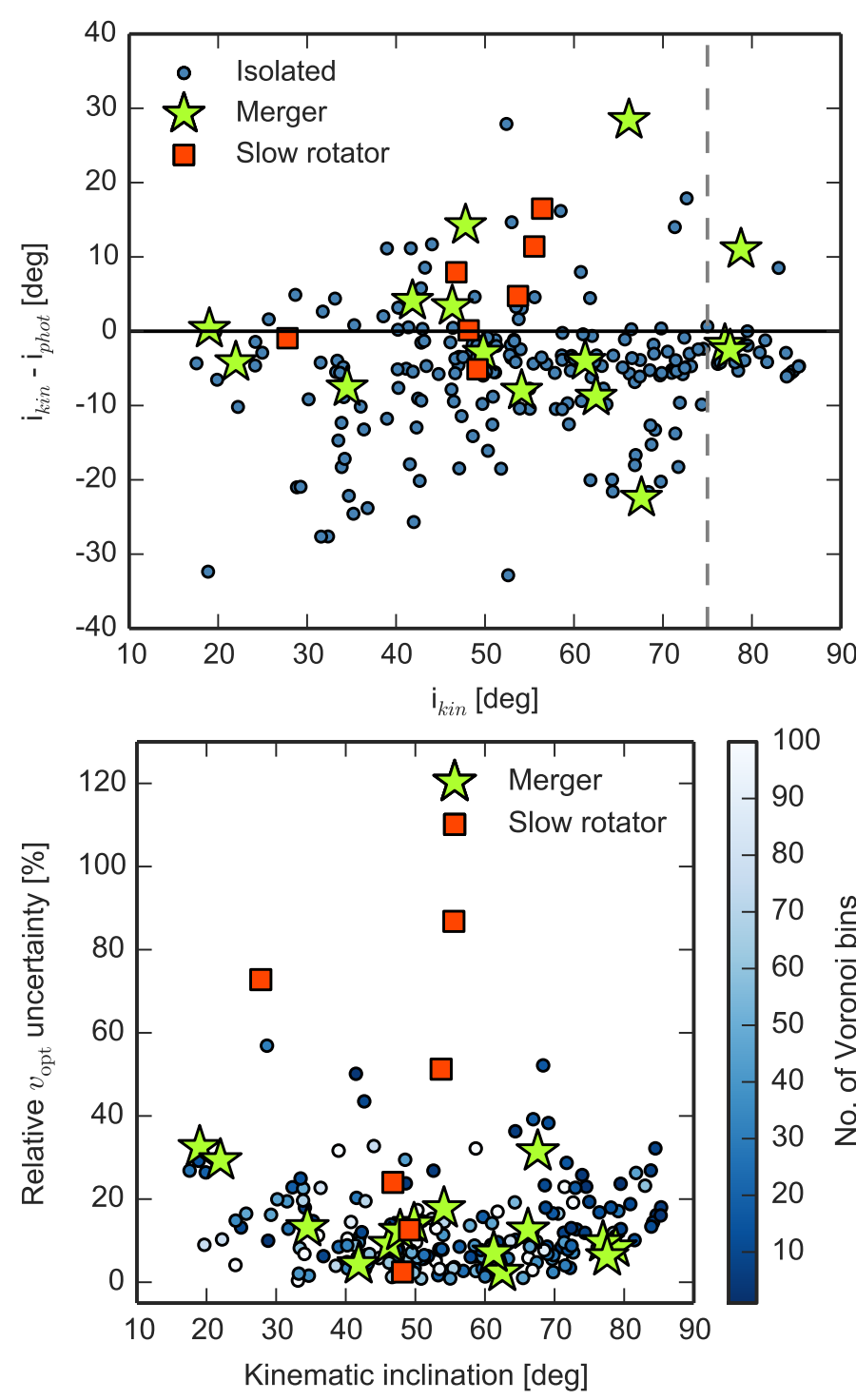

Fig. 10. Top: difference between kinematic and photometric inclination estimates, calculated from $r$-band axis ratios assuming the intrinsic disk thickness $q=0.2$. Slow rotators and interacting galaxies are marked in red and light green, respectively. Bottom: $v_{\text {opt }}$ uncertainties vs. kinematic inclinations.

instance, mergers and slow rotators showing little or no ordered rotation could not be well constrained and had broad, nonGaussian posterior distributions of $v_{2.2}$. Relative $v_{\text {opt }}$ uncertainties vs. kinematic inclinations are shown in Fig. 10, demonstrating the difficulty of constraining the rotation velocity of slow rotators. Because of their large velocity uncertainties, they would not contribute significantly to the fit of the TFR. However, the majority of such galaxies were rejected from the final TullyFisher sample as described in Sect. 5.2.

Other potential sources of uncertainties include the limited CALIFA field of view and the spatial resolution of the binned stellar velocity fields. However, they are either implicitly included in the posterior $v_{\mathrm{opt}}$ uncertainties or too difficult to estimate within the scope of this paper. A careful analysis of uncertainties in the template TFR context is presented in Saintonge \& Spekkens (2011).

In most previous TF work the photometric axis ratio $b / a$ has been directly converted into inclination, without assuming any associated uncertainties or potential difference between kinematic inclination (i.e. the real inclination of the observed rotating component) and its photometric estimate based on $b / a$. This has led to rotation velocity estimates with uncertainties of the order of a few $\mathrm{km} \mathrm{s}^{-1}$ and TFRs with negligible uncertainties on the line fit parameters. In our opinion, using kinematic inclination values is more justified than deriving them independently from the photometric axis ratio. In addition, MCMC modelling of the velocity fields provides the full posterior distribution of velocity uncertainties. This consistent self-contained rotation curve modelling is only possible with IFS data.

However, the stellar velocity fields we used here have their own share of problems, such as large Voronoi bins and limited spatial extent, and therefore are likely to lead to larger velocity measurement uncertainties than the other methods. A direct comparison on the velocity uncertainties derived using the different methods and data (such as stellar or gas 2D velocity fields or long-slit observations) is beyond the scope of this paper. The consistent velocity uncertainties provided by the methods presented here can be propagated into a TFR fit, providing more reliable constraints on its internal scatter.

\subsection{Calculating the circular velocity}

We used stars as the tracer of the circular velocity of the galaxies. Stellar velocity fields have the advantage of being available for all morphological types and also of being less distorted than gas fields (Adams et al. 2012; Kalinova \& Lyubenova 2016). However, stars are dynamically hot tracers, and a socalled asymmetric drift correction, which takes the velocity dispersion into account, is frequently applied to obtain the circular velocity $v_{\text {circ }}$.

According to Kalinova \& Lyubenova (2016) and Kalinova et al. (2016), the classical (Weijmans et al. 2008) asymmetric drift correction (ADC) underestimates the real underlying potential if the local inclination-corrected rotation velocity and velocity dispersion ratio $V / \sigma$ is lower than 1.5 . This implies that asymmetric drift corrections would be inaccurate for the majority of galaxies within our sample. Even though the asymmetric drift correction changes the shape of the inner rotation curve dramatically, the estimated circular velocity does not change significantly for rotation-supported galaxies. However, the classical ADC approach is unsuitable when the assumption of a thin disk is not valid.

We decided to avoid the classical ADC to treat our sample in a consistent, homogeneous manner. Although advanced dynamical modelling is beyond the scope of this observational paper, we decided to apply an empirical correction based on the findings of Kalinova \& Lyubenova (2016). They analysed the difference between dynamical masses inferred using classical ADC models and axisymmetric Jeans anisotropic multi-Gaussian (JAM) models applied to stellar mean velocity and velocity dispersion fields of 18 late-type galaxies observed with the SAURON IFS instrument. We used the relation derived from Table 4 of Kalinova et al. (2016) and calculated the circular velocities by multiplying the measured velocity by the square root of the factors provided, based on the local measured $v_{\text {opt }} /$ LOS $\sigma_{\text {opt }}$ of a galaxy. The uncertainty of the calculated circular velocity was calculated by adding in quadrature $10 \mathrm{~km} \mathrm{~s}^{-1}$ multiplied by $1+\Delta V$ (where $\Delta V$ is the square root of the uncertainty factor from Kalinova et al. 2016) to the $v_{\text {circ }}$ uncertainty budget. The magnitude of the correction is shown in Fig. 11.

We compared the gas rotation velocities of 44 galaxies obtained from CALIFA DR1 data (García-Lorenzo et al. 2015) with their stellar circular velocity values, shown in Fig. 12 below. 


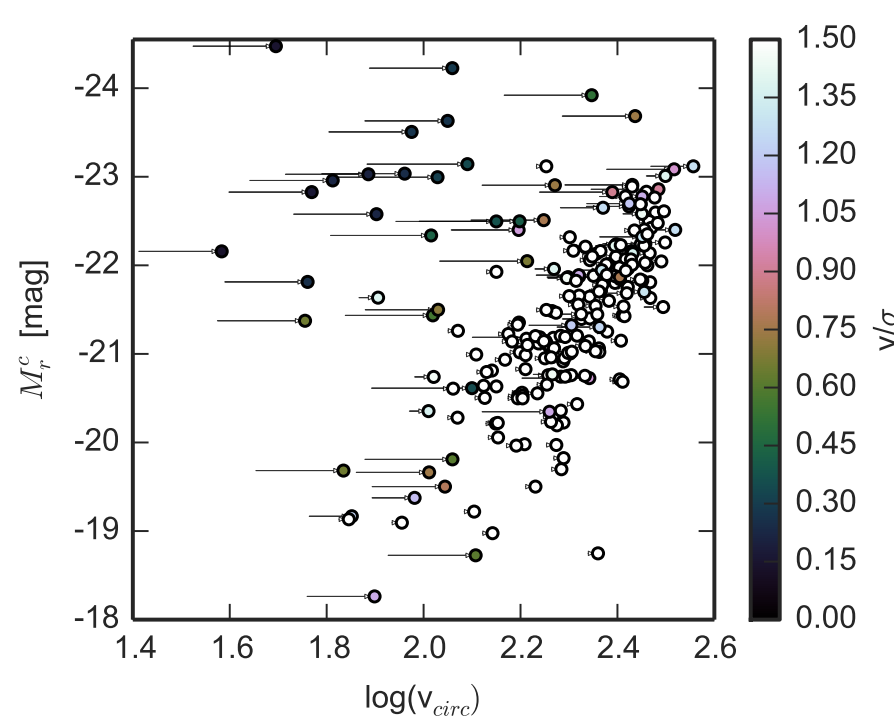

Fig. 11. Magnitude of the circular velocity correction shown as arrows. The points are the final circular velocity values, colour-coded for the $v_{\text {opt }} / \sigma$ factor. The galaxy with the lowest $\log \left(v_{\text {circ }}\right)$ value, a slow rotator, is not shown for clarity. This galaxy, NGC $6515\left(\log \left(v_{\text {circ }}\right)=1.05\right)$, was excluded from further analysis during the outlier rejection procedure described in Sect. 5.2.

Ionised gas rotation curves were obtained from the envelope of the position-velocity diagram and corrected for inclination using photometric axis ratio $b / a$, then gas $v_{\text {opt }}$ values were evaluated at the optical radius.

This is not a direct and accurate comparison for many reasons. First of all, even though ionised gas is typically less dynamically hot than the stars, the measured gas rotation velocity does not trace the gravitational potential directly and the gas dispersion needs to be taken into account. This presents additional difficulties because the gas dispersion cannot be measured directly with the CALIFA spectral resolution. Furthermore, thermal motion and gas turbulence also contribute to the total gas velocity dispersion and cannot be distinguished from gravitationally induced velocity dispersion without additional data (Weijmans et al. 2008). In addition, the gas $v_{\text {opt }}$ was estimated in a different way from the stellar rotation velocity. Photometric inclination estimates were used instead of kinematic ones and no rotation curve modelling was performed, which means that any warps or distortions present in ionised gas were not accounted for. The most noticeable outliers in Fig. 12 are offset as a result of the latter reasons. Despite this, the two quantities are close to each other for most of the galaxies, with the stellar $\mathrm{v}_{\text {circ }}$ being typically larger as expected from the arguments above. A similar comparison for several CALIFA galaxies, using a different asymmetric drift correction method, is shown in Aguerri et al. (2015).

We use the calculated circular velocity values in all further analysis unless noted otherwise.

\section{Separation of different populations of galaxies in the $\boldsymbol{M}_{r}-\boldsymbol{v}_{\text {circ }}$ plane}

\subsection{Specific angular momentum}

The specific angular momentum $j$ and the total mass are key properties of galaxies that strongly influence their morphology, luminosity, and secular evolution. A directly measurable quantity in IFS observations, related to $j$, is the $\lambda_{R}$ parameter, defined

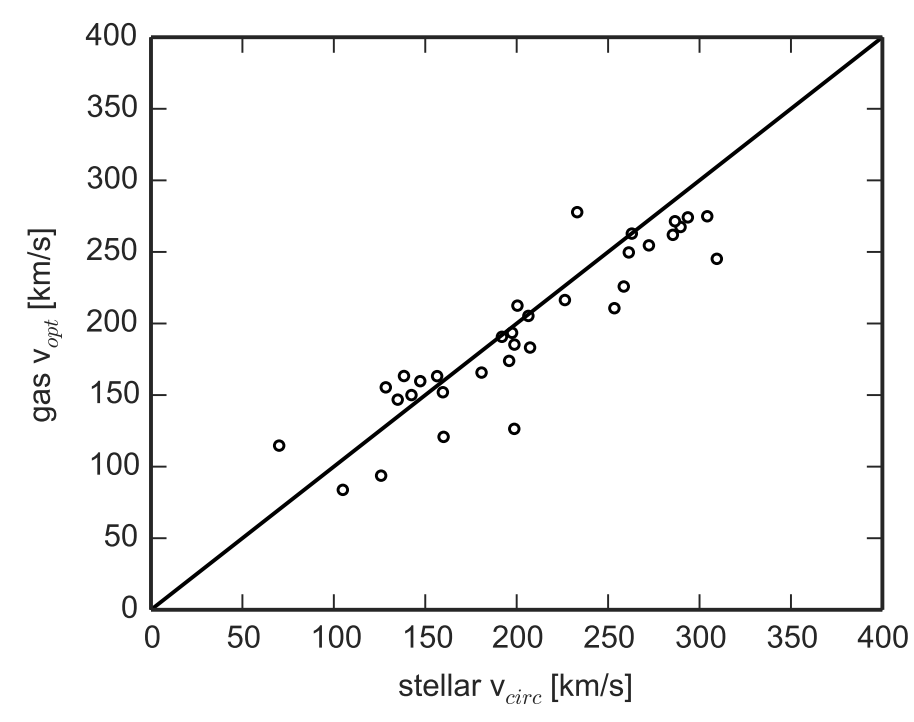

Fig. 12. Comparison between ionised gas rotation velocity at the optical radius and the stellar circular rotation velocity for 44 CALIFA DR1 galaxies.

in Emsellem et al. (2007) as

$\lambda_{R} \equiv \frac{\langle R|V|\rangle}{\left\langle R \sqrt{V^{2}+\sigma^{2}}\right\rangle}$.

In practice, $\lambda_{R}$ is calculated in the following way (Emsellem et al. 2007):

$$
\lambda_{R}=\frac{\sum_{i=1}^{N_{p}} F_{i} R_{i}\left|V_{i}\right|}{\sum_{i=1}^{N_{p}} F_{i} R_{i} \sqrt{V_{i}^{2}+\sigma_{i}^{2}}},
$$

where $F_{i}, R_{i}, V_{i}$ and $\sigma_{j}$ are the fluxes, semi-major axis values, velocities, and velocity dispersion values of a spatial Voronoi bin $i$.

Measurements of $\lambda_{R}$ parameter values are available for the galaxies in our sample from work done within the CALIFA team. For CALIFA galaxies, the $\lambda_{\text {Re }}$ parameter $\left(\lambda_{R}\right.$ within one effective radius $R_{\mathrm{e}}$ ) was calculated as described by Eq. (5) and corrected for inclination as described in Falcón-Barroso et al. (2016), also see Falcón-Barroso et al. (2015) and Querejeta et al. (2015). Briefly, ellipticities $\epsilon$ were obtained from IRAF ellipse fit models of the SDSS $r$-band images, and the probability of observing a galaxy with an inclination $i$, given its ellipticity $\epsilon$, was calculated as

$f(i \mid \epsilon)=\frac{f(q)(1-\epsilon)}{\sqrt{\sin ^{2} i-\epsilon(2-\epsilon)}}$,

where $f(q)$ is the intrinsic shape distribution of galaxies. The $\lambda_{\operatorname{Re}}$ values were available for 206 out of 226 galaxies because the authors rejected the galaxies with a low number of bins (typicaly $\leq 10)$ and interacting galaxies showing obvious kinematic irregularities from their calculation.

Although minor inconsistencies arise from using slightly different parameters (such as inclinations) in this analysis, we emphasize that the lambda parameter was used in this study only as a qualitative illustration of the degree of rotation support in our sample galaxies. These minor inconsistencies and lack of $\lambda_{\operatorname{Re}}$ values for some of the galaxies thus have no influence on any quantitative result in the paper. 


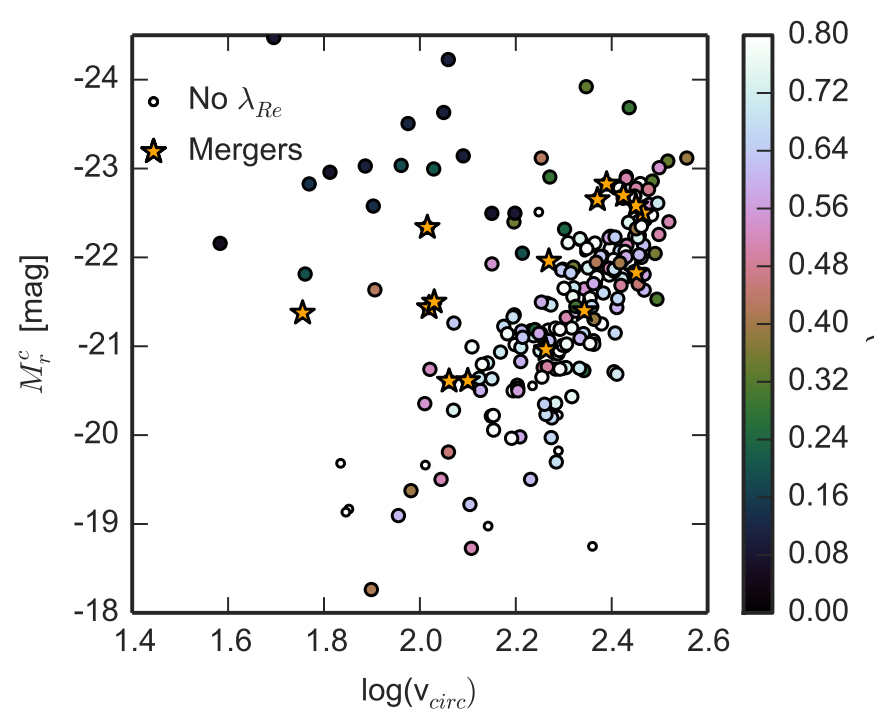

Fig. 13. Galaxies on the $v_{\text {circ }}-M_{r}^{c}$ plane, colour-coded for inclinationcorrected $\lambda_{\mathrm{Re}}$ parameter. The 20 galaxies without $\lambda_{\mathrm{Re}}$ values available (see text) are shown as smaller white circles.

Figure 13 shows that the galaxies on the circular velocityluminosity plane are drawn from at least two parent distributions: galaxies that exhibit significant ordered rotation and belong on the TFR, and the rest, including pressure-supported galaxies and some ongoing mergers. As a consequence, some of the data, even though they are of reasonable quality, are simply beyond the scope of the simple model of the TFR, which is a linear relation with small intrinsic scatter. Since linear regression is very sensitive to outliers and, more importantly, some of the galaxies in our sample do not belong on the TFR by definition, some sort of outlier rejection must be performed.

\subsection{Modelling the TFR as a mixture of Gaussians}

We did not apply any additional selection criteria to our galaxy sample (see Sect. 2), except for those that are implicit in the CALIFA mother sample selection and properties of the SDSS survey. As a result, it contains different galaxy populations, not all of which are well described by a thin rotating-disk model assumed in Sect. 4 (mergers and slow rotators are two examples).

We did not simply reject the outliers using an arbitrary procedure such as hand-pruning the data, sigma-clipping, or straightforward rejection of slow rotators and visually classified mergers. Instead, we modelled the distribution as a mixture of data obtained from two different generative models: a narrow, linear relation with Gaussian noise and small intrinsic scatter (corresponding to the subset of galaxies to which the TFR applies) and a two-dimensional Gaussian distribution that includes the galaxies that lie farther away from the linear relation.

The probability density function of a linear TFR with a small intrinsic Gaussian scatter $\sigma_{i}$ is

$$
\begin{aligned}
P(v \mid M, m, b, \sigma)= & \frac{1}{\sqrt{2 \pi\left(\sigma_{y}^{2}+m^{2} \sigma_{x}^{2}+\sigma_{i}^{2}\right)}} \\
& \times \exp \left[-\frac{(M-m v-b)^{2}}{2\left(\sigma_{y}^{2}+m^{2} \sigma_{x}^{2}+\sigma_{i}^{2}\right)}\right],
\end{aligned}
$$

where $v$ is the logarithm of circular velocity, $M$ is the absolute magnitude, and $m$ and $b$ are the slope and the offset of the linear relation.
The non-TF distribution is quite sparse, therefore we chose a non-restrictive two-dimensional Gaussian model described by its mean in two dimensions $\left(\mu_{x}, \mu_{y}\right)$ and a covariance matrix

$\Sigma=\left|\begin{array}{cc}\sigma_{\text {bad }_{\mathrm{x}}}^{2} & \rho \sigma_{\mathrm{bad}_{\mathrm{x}}} \sigma_{\mathrm{bad}_{\mathrm{y}}} \\ \rho \sigma_{\mathrm{bad}_{\mathrm{x}}} \sigma_{\mathrm{bad}_{\mathrm{y}}} & \sigma_{\mathrm{bad}_{\mathrm{y}}}^{2}\end{array}\right|$.

Here $\sigma_{\mathrm{bad}_{x, y}}$ are the standard deviations of the non-TF points population, whose shape is allowed to vary, and $\rho$ is its correlation term.

When we combine both models, we can obtain a probability of belonging to the TFR for each datapoint and reject the outliers based on this probability. We finally had seven free parameters describing the two distributions ( $\left.m, b, \sigma_{i}, \mu_{x}, \mu_{y}, \sigma_{\text {bad }_{x}}, \sigma_{\text {bad }_{\mathrm{y}}}\right)$, which we inferred and marginalised over the nuisance parameters $P_{b}$ (the probability of any point belonging to the non-TF distribution) by finding their posterior distributions using MCMC. The log-likelihood of the mixture of the two distributions described above is

$$
\begin{aligned}
& \ln \mathcal{L} \propto \\
& -0.5 \Sigma\left(\left(1-P_{b}\right) \cdot\left[\ln \left(\sigma_{y}^{2}+m^{2} \sigma_{x}^{2}+\sigma_{i}^{2}\right)+\frac{(y-m x-b)^{2}}{\left(\sigma_{y}^{2}+m^{2} \sigma_{x}^{2}+\sigma_{i}^{2}\right)}\right]\right. \\
& \left.+P_{b} \cdot\left[\frac{\left(x-\mu_{x}\right)^{2}}{\left(\sigma_{x}^{2}+\sigma_{\text {bad }_{\mathrm{x}}}^{2}\right)}+\frac{\left(y-\mu_{y}\right)^{2}}{\left(\sigma_{y}^{2}+\sigma_{\text {bad }_{\mathrm{y}}}^{2}\right)}\right]\right) .
\end{aligned}
$$

Modelling involves setting priors on several of the parameters. The sparsity of the population offset from the TFR and because we worked with the logarithm of velocity, which skews the error distribution, meant that we applied Gaussian priors on its mean and variance, based on the estimated moments of the slow rotators population. We also applied a wide Gaussian prior, based on a simple linear fit to the fast rotators alone, on the slope $m$, and, naturally, limited the $P_{b}$ to be between 0 and 1 and $\sigma_{i}, \sigma_{\text {bad }}>0$.

The results of the mixture modelling are shown in Fig. 14. We rejected the datapoints with likelihoods lower than 1 $P_{\text {good }}=0.5$, which were those that more likely belong to the non-TFR distribution. This resulted in the rejection of 27 galaxies that were not compatible with being on a linear relation. The remaining 199 galaxies were used in the further analysis and are named the TF sample.

\subsection{Properties of the outlier galaxies}

Seven out of 27 rejected galaxies are slow rotators with $\lambda_{\mathrm{Re}}<0.1$ (Fig. 15). Two galaxies are classified as mergers, and several of the rejected galaxies are not sufficiently sampled by the CALIFA observations.

A comparison of absolute magnitudes and morphologies between the useful sample (described in Sect. 2) and the resulting TF and non-TF samples yielded by the mixture of Gaussian modelling is shown in Fig. 16. The most salient property of the outlier rejection is the removal of the majority of bright earlytype ellipticals from the TF sample, which has a clear physical basis because such galaxies are much more likely to be slow rotators. However, even if this is expected, the rejection was based not on visual classification, but driven by the data, meaning that it was based on the location of the galaxy on the $M_{r}^{c}-v_{\text {circ }}$ plane and the uncertainties. 


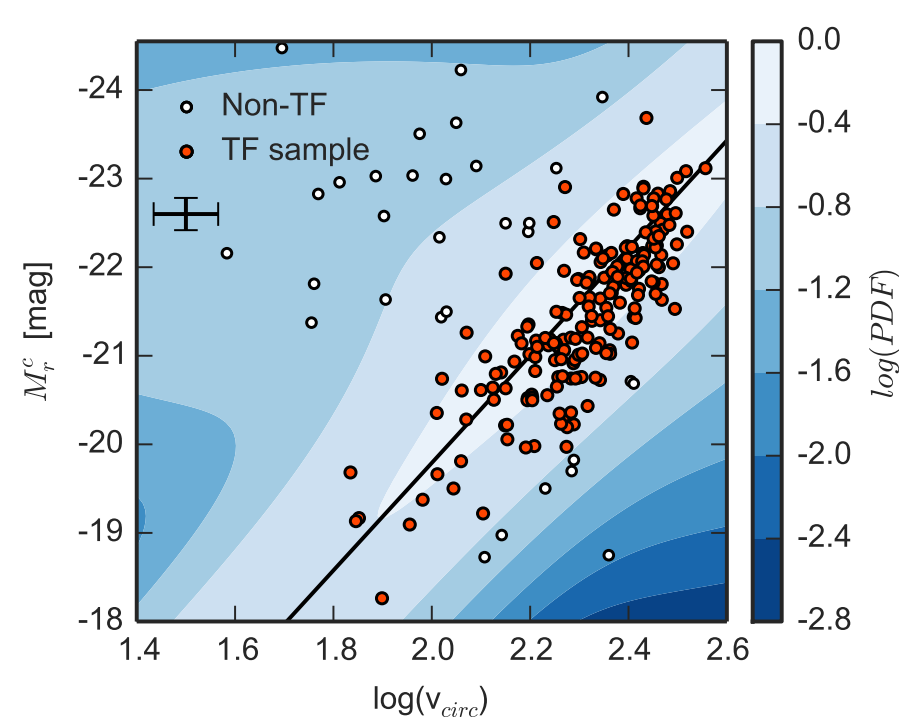

Fig. 14. Distribution of non-TF points (white) and the TF population (red), together with their underlying estimated generative functions (a 2D Gaussian and a linear relation with small intrinsic scatter), as described in the text. Error bars in the upper left corner show the mean uncertainties in $M_{r}^{c}$ and $\log \left(v_{\text {circ }}\right)$.
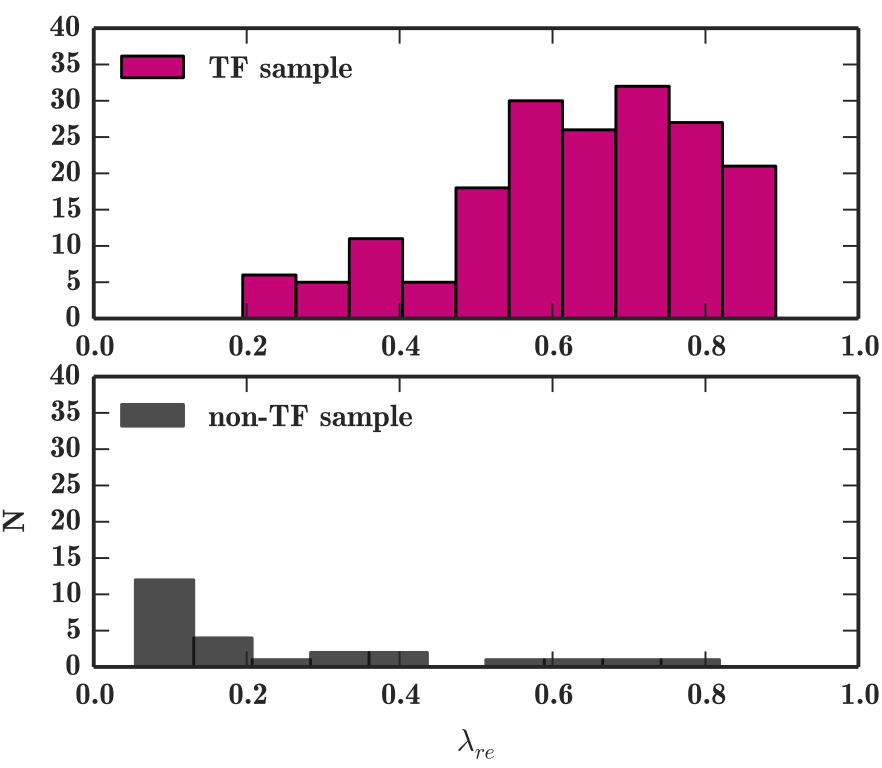

Fig. 15. Lambda parameter $\lambda_{\mathrm{Re}}$ histogram for the TF sample (top) and the 25 outlier galaxies for which $\lambda_{\mathrm{Re}}$ values were available.

\subsection{Properties of the Tully-Fisher sample}

To characterise the final TF sample, we compared the luminosity functions derived from the volume-corrected CALIFA mother sample and the volume-corrected TF sample (Fig. 2). We performed the procedure as described in Sect. 2 and W14: by weighting each galaxy with its $1 / V_{\max }$ factor.

They differ significantly at the lower luminosity end, where the LF of the TF sample falls off sharply. The difference is not as pronounced for the brightest galaxies because of the low number statistics for such objects in the volume-complete sample. We did not a priori expect to retain the volume completeness during the outlier rejection because the rejection is non-random. The difference between the luminosity functions is an expected outcome of the outlier rejection procedure described in Sect. 5.2.
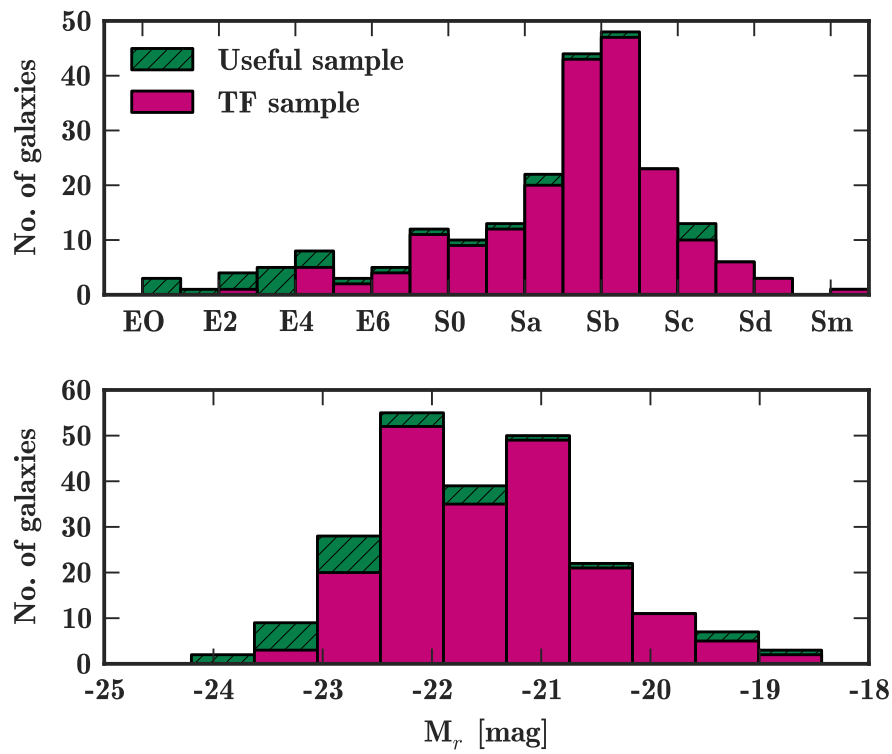

Fig. 16. Morphological type and absolute magnitude histograms of the final TF sample and the useful sample.

However, the mixture modelling is a reproducible procedure. Given a statistically representative sample, the same procedure can be performed again, yielding a distribution of galaxies that is representative of the overall rotation-supported galaxy population and is a subset of the joint volume-complete sample (within the limits of the observed sample). Even so, Fig. 2 shows that the Tully-Fisher sample can nevertheless be considered to be volume complete-able within the $-20 \geq M_{r}^{c} \geq-22$ mag range.

To verify that velocity-field-based measurements do not show systematic offsets from the conventional long-slit measurements, we show the $M_{r}^{c}-v_{\text {circ }}$ distribution of the TF sample and a comparison sample from Courteau (1997, hereafter C97) in Fig. 17. The TF sample shows a larger scatter than the comparison sample (the rms error of $\log \left(v_{\text {circ }}\right)$ is equal to $0.26 \mathrm{dex}$ ), which is expected given that the comparison sample used a sample of galaxies with specific selection criteria, such as late Hubble types, moderately high inclination, and lack of interactions or peculiar properties (Courteau 1996). The TF sample has a higher proportion of brighter galaxies of earlier types. In addition to this, the CALIFA stellar circular velocities are typically higher than $\mathrm{H} \alpha v_{\text {opt }}$ measurements as a consequence of the circular velocity correction. A direct comparison would involve calculating an equivalent correction for the gas, which is not a trivial endeavour, as discussed in Sect. 4.4. Nevertheless, the plot shows that circular velocity measurements from IFS velocity fields and rotation curves are compatible and do not show a significant systematic deviation from a similar underlying relation.

\section{Tully-Fisher relation}

Even though a simple straight-line model is not accurate, it is a useful tool in the area of distance determination and has been widely used to model the TFR, as well as to compare the local relation with high-redshift galaxy samples.

For comparison purposes we fitted the TFR as a straight line with free slope, intrinsic scatter and offset (zeropoint) parameters $m, \sigma_{i}, b$, assuming $M_{r}^{c}$ as the independent variable. We used the HYPER-FIT hyperplane fitting package (Robotham \& Obreschkow 2015), which provides the tools to fit heteroscedastic and covariant data, and used $1 / V_{\max }$ values as the fit weights. 


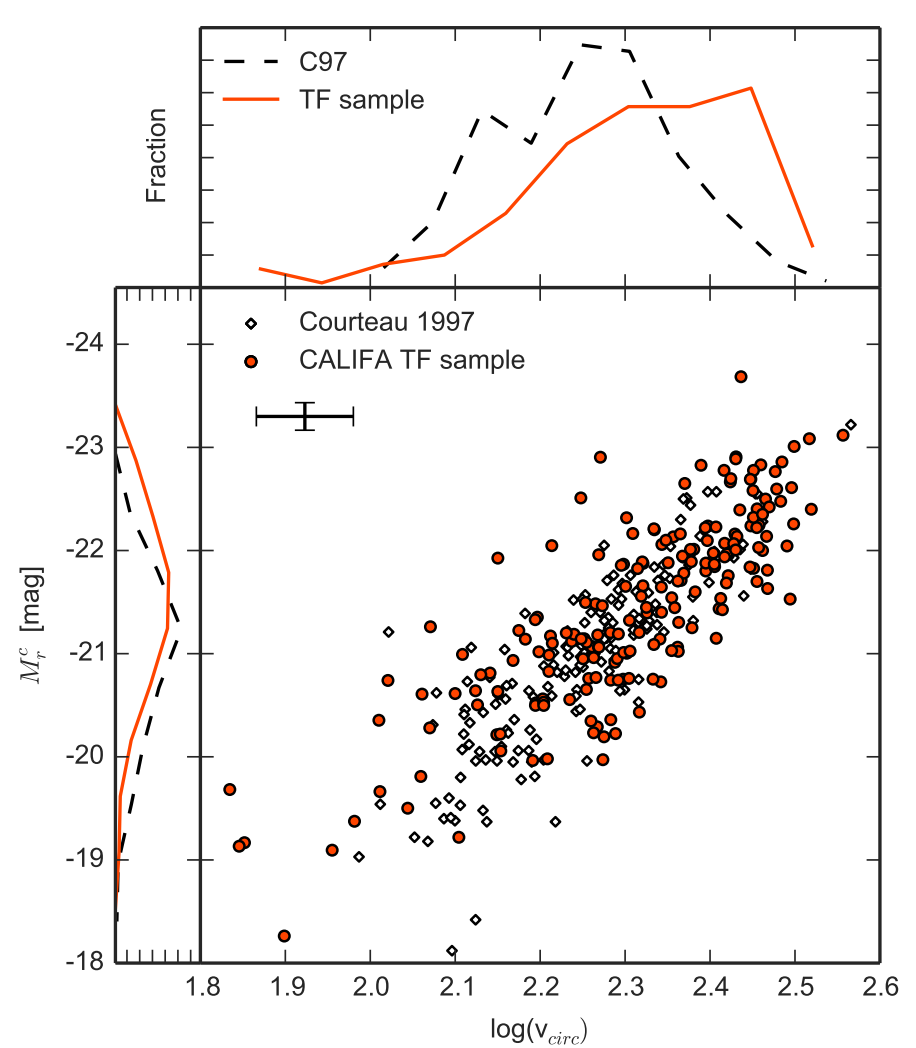

Fig. 17. Comparison of our Tully-Fisher sample $M_{r}^{c}-v_{\text {circ }}$ distribution and Courteau (1997, C97) $M_{r}^{c}-\mathrm{H} \alpha v_{\text {opt }}$ measurements. The marginal plots show normalised histograms for both samples. The mean uncertainty magnitudes of CALIFA measurements are shown by the error bars.

To verify that the limited spatial coverage of CALIFA velocity fields and the necessity of extrapolating some rotation curves beyond the last measured point (see Sect. 2) did not introduce a bias, we checked where our datapoints lie on the Tully-Fisher relation. There is no significant offset (Fig. 18).

We also verified that the infall corrections described in Sect. 3 did not affect the absolute magnitudes significantly by comparing them with luminosities estimated using pure Hubbleflow-based distances. Although the average difference between the two values is equal to $0.14 \mathrm{mag}$, there is no systematic effect on the TFR. Similarly, the lack of the extinction corrections described in Sect. 3 had a negligible effect on the intrinsic scatter of the TFR, but it made the slope slightly steeper.

We compared our fit result with C97 and Pizagno et al. (2007, hereafter P07), who investigated the $r$-band TF relation using $\mathrm{H} \alpha$ rotation curves, based on $v_{\text {opt }}$. Our TFR, fit using a $v_{\text {circ }}$ estimate, is shifted to the right and is steeper. Using the $1 / V_{\max }$ weights leads to a slightly flatter TFR. This is expected because the unweighted relation is dominated by the more luminous galaxies. Applying volume weights acts in the opposite direction and brings the fit relation closer to the results of C97, which are based on a sample dominated by late-type spirals, as shown in Fig. 17.

The slope, offset, and scatter values for a $v_{\text {circ }}$ and $v_{\text {opt }}$-based Tully-Fisher relation are provided in Table 1 . This table also contains the forward TFR parameters provided by C97 and $\mathrm{P} 07$. We note that the scatter value reported by us is not the standard deviation of the points from the straight fit line, but the intrinsic scatter not accounted for by the measurement uncertainties during the modelling. The rms error of our measurements is 0.26 mag.

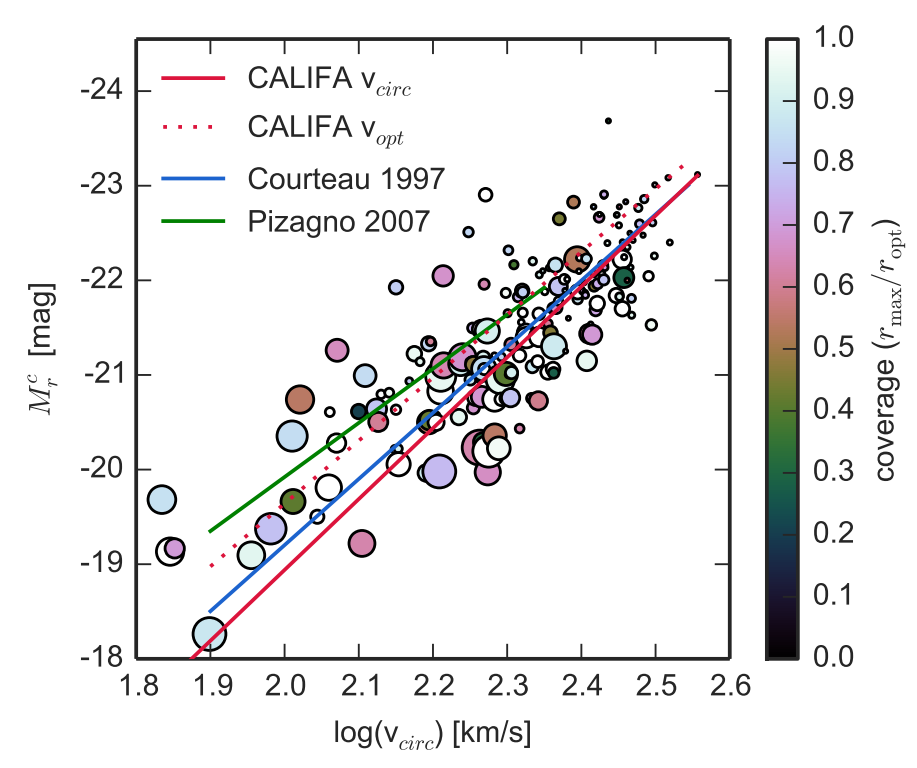

Fig. 18. Linear fit (see text for more details) to CALIFA $v_{\text {circ }}-M_{r}^{c}$ data, and comparison with Courteau (1997) and Pizagno et al. (2007) TF fits. Point sizes are proportional to $1 / V_{\max }$ weights, colours indicate insufficient spatial coverage of the velocity field (sampling of the rotation curve is lower than $1 r_{\mathrm{opt}}$ ). The fit to $v_{\mathrm{opt}}-M_{r}^{c}$ is also shown in the plot.

Table 1. Tully-Fisher relation fit parameters and literature values.

\begin{tabular}{cccc}
\hline \hline Velocity definition & Slope & Offset & Scatter \\
\hline CALIFA $v_{\text {circ }}$ & $-7.5 \pm 0.5$ & $-4.0 \pm 1.0$ & $0.03 \pm 0.06$ \\
CALIFA $v_{\text {opt }}$ & $-6.7 \pm 0.4$ & $-6.3 \pm 0.9$ & $0.09 \pm 0.03$ \\
C97 $v_{\text {opt }}$ & $-6.99 \pm 0.33$ & $-5.23 \pm 0.46$ & 0.46 \\
P07 $v_{\text {opt }}$ & $-5.72 \pm 0.19$ & $-7.9 \pm 0.03$ & 0.42 \\
\hline
\end{tabular}

The intrinsic scatter value we obtain suggests low upper limits on the intrinsic scatter of the TFR. The sources of it include scatter in the dark matter halo spin, concentration and response to galaxy formation (Dutton et al. 2011), potential ellipticity (Franx \& de Zeeuw 1992) and formation history (Eisenstein \& Loeb 1996; Giovanelli et al. 1997), mass-to-light ratio (Gnedin et al. 2007) and morphology (Giovanelli et al. 1997), among others. A more in-depth study of the intrinsic TFR scatter that would include the additional measurement errors resulting from our adopted infall, extinction and circular velocity corrections is beyond the scope of this paper, but it is unlikely that the reported intrinsic scatter would be increased.

\section{Volume-corrected bivariate distribution function in the Tully-Fisher plane}

The volume and large-scale correction procedure described in Sect. 2 can be used to reconstruct a volume-complete bivariate distribution in the $M_{r}^{c}-v_{\text {circ }}$ plane, applicable within the CALIFA completeness limits.

We used the kernel density estimation (KDE) to achieve this. KDE is a non-parametric probability density estimation procedure (Rosenblatt 1956; Parzen 1962) consisting of representing each datapoint as a smooth distribution and then inferring the underlying density distribution. It is superior to histograms because a smooth kernel can be chosen, there is no dependence on the choice of the starting bin, and the probability density function is obtained naturally. 

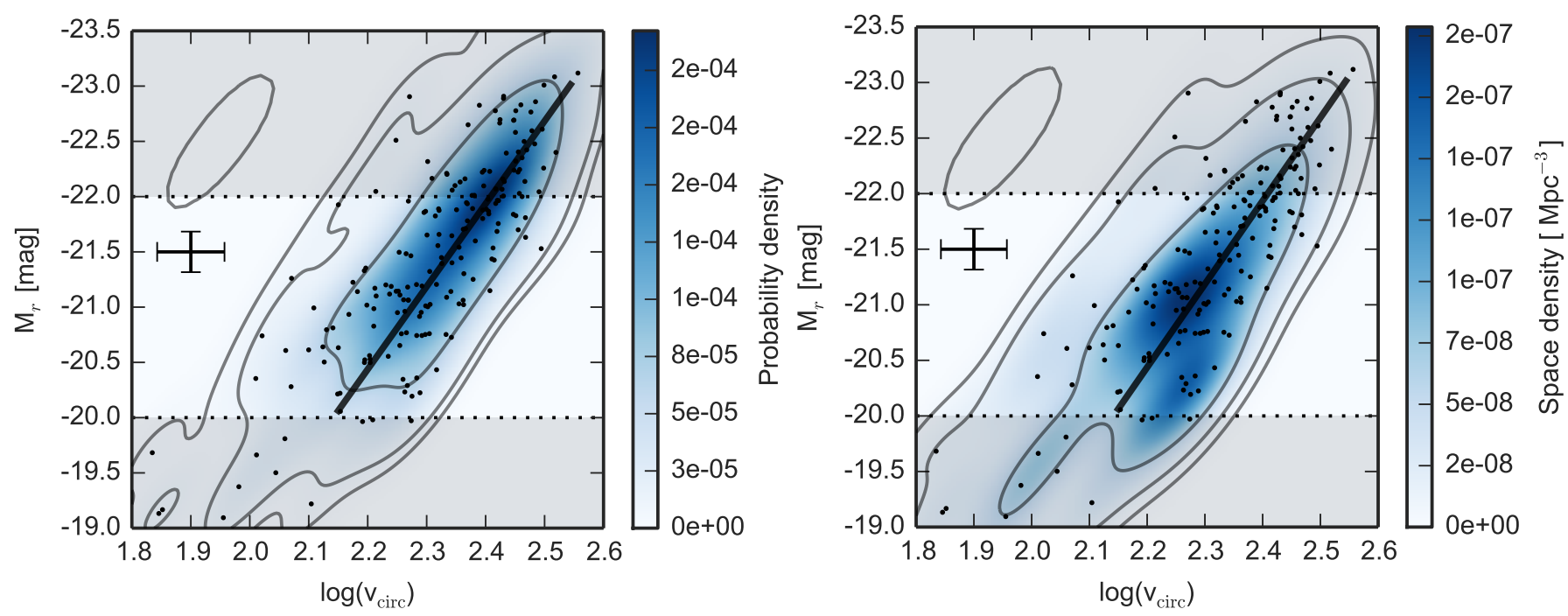

Fig. 19. Left plot: joint probability density of $M_{r}^{c}-v_{\text {circ }}$. Right plot: joint space densities distribution, estimated by weighting the KDE kernels by the $1 / V_{\max }$ weights. Grey lines denote the 1,2 , and 3 standard deviation contours. The black line shows the TFR discussed in the previous section. A $1 \sigma$ contour of the Gaussian kernel used for density estimation is shown in the upper left corners. Grey shaded regions mark the regions where our sample is incomplete.

We chose a two-dimensional Gaussian as the kernel function. The size and orientation (the kernel function covariance matrix) can be selected using various methods, such as cross-validation, or using one of the rules-of-thumb that empirically estimate the bandwidth based on the number of data points and dimensions of the dataset. We estimated the optimal kernel bandwidth based on the global shape of the distribution and the Silverman rule (Silverman 1986). First of all, the global covariance matrix of all the observed points was estimated, assuming that the observed point distribution is similar to a Gaussian distribution in a sense that it is unimodal, symmetric, and not heavy-tailed. Then this matrix was multiplied by a scaling factor $f_{s}=n^{-\frac{1}{6}}$, derived according to the Silverman rule (Scott \& Sain 2005). Here $n$ is the number of data points.

We used the stats.gaussian_kde routine from SciPy package (Jones et al. 2001-2015) as the basis for our analysis. The left panel of Fig. 19 shows the probability density distribution in the $v_{\text {circ }}-M_{r}^{c}$ space. The density distribution here is dominated by the brighter galaxies with $M_{r}^{c}>-20.5 \mathrm{mag}$ and $v_{\text {circ }}>200 \mathrm{~km} \mathrm{~s}^{-1}$ because of the CALIFA sample construction and smaller relative scatter at $\operatorname{larger} \log \left(v_{\text {circ }}\right)$ values.

However, this picture changes when the $1 / V_{\max }$ factors are included as additional KDE weights, as shown in the right panel of Fig. 19. The area with the highest probability density now shifts to lower velocities $\left(<250 \mathrm{~km} \mathrm{~s}^{-1}\right)$ and magnitudes $\left(M_{r}^{c}>\right.$ $-21.5 \mathrm{mag}$ ). We converted the probability density to space densities by multiplying the probability density (which integrates to 1 ) by the sum of all the $1 / V_{\max }$ factors in the TF sample.

The joint distribution of the luminosity function and the velocity function (discussed in an upcoming paper) are able to constrain galaxy formation and evolution models more than a single marginal distribution (LF or VF). The linear TFR does not directly provide information about the number of galaxies at a given location in the $M_{r}^{c}-v_{\text {circ }}$ plane, whereas we provide space densities that can be compared with simulations of cosmological volumes.

When comparing a model or simulation of a galaxy with the TFR, this traditionally consists of ensuring that the produced galaxies lie on the TFR. The TFR is typically defined by the slope and offset parameters, in some cases including the scatter.
Here we determined the full volume-corrected bivariate distribution, or probability distribution, in the $L-v_{\text {circ }}$ plane for the first time. This allows a much more direct and quantitative estimate of the likelihood that a simulated galaxy is consistent with the real galaxy population. The halo velocities obtained from cosmological simulations would have to be converted into the circular velocity, which can be directly compared with our results. Analysis of such a volume-complete distribution, which would require a larger sample spanning diverse environments and luminosities, however, might shed some light on the environmental influence on the TFR, as indicated by Blanton et al. (2008).

Our analysis is limited by incompleteness problems at the low-velocity or fainter magnitude end. Nevertheless, the difference in the two distributions is apparent, showing that the most luminous galaxies do not contribute significantly to the bulk of the Universe's stellar angular momentum. The space densities shown in Fig. 19, as well as the other data, are available online at CDS.

\section{Conclusions}

We presented the first space density distribution of rotating galaxies in the $M_{r}^{c}-v_{\text {circ }}$ plane, derived using the CALIFA stellar velocity fields. The use of stellar IFS kinematics, careful extinction corrections, and the statistically well-understood CALIFA sample allowed us to perform volume corrections and provided a fair representation of the distribution of galaxies with $-20>$ $M_{r}^{c}>-22$ mag. Our key results are as follows.

- The velocity uncertainties in many TFR analyses are underestimated. The reason for this is a combination of direct use of photometric inclination estimates, lack of full 2D spatial information, and degeneracies between rotation curve parameters and inclination. Using consistent MCMC modelling of velocity fields, we obtained realistic velocity uncertainties, which were propagated to the further analysis.

- By avoiding any arbitrary cuts in our sample and instead modelling the TFR and non-TFR populations of galaxies, we used a reproducible, probabilistic approach to outlier rejection (Sect. 5.2). This allowed us to generalise our analysis to 
other samples and let us preserve the capability of performing volume corrections.

- A $1 / V_{\max }$-weighted linear fit with bivariate uncertainties provided an $r$-band TFR with slope, zeropoint and scatter equal to $-7.5,-4.0,0.03$ for the $v_{\text {circ }}$-based TFR and $-6.7,-6.3$ and 0.09 for $v_{\text {opt }}$-based TFR (Sect. 6).

- We provided a bivariate local space density distribution in the $v_{\text {circ }}-M_{r}^{c}$ plane (Sect. 7), which, although less straightforward to compare with than a simple linear parameterisation, provides more information than a single line and is more representative of the overall properties of galaxies. The full 2D distribution is what simulations ought to be compared with.

Acknowledgements. We sincerely thank S. Courteau and the anonymous referee for carefully reading the draft, thoughtful suggestions, and providing us with an opportunity to improve the manuscript. S.B. acknowledges financial support from BMBF through the Erasmus-F project (grant number 05 A12BA1). C.J.W. acknowledges support through the Marie Curie Career Integration Grant 303912. JFB. acknowledges support from grant AYA2013-48226-C3-1$\mathrm{P}$ from the Spanish Ministry of Economy and Competitiveness (MINECO) IM acknowledges financial support from the Spanish Ministry of Economy and Competetiveness through the grant A\&A2013-0422277. This study makes uses of the data provided by the Calar Alto Legacy Integral Field Area (CALIFA) survey (http://califa.caha.es/). It is based on observations collected at the Centro Astronómico Hispano Alemán (CAHA) at Calar Alto, operated jointly by the Max-Planck-Institut fúr Astronomie and the Instituto de Astrofisica de Andalucia (CSIC). In addition to that, we used the data from SDSS DR7 (Abazajian et al. 2009). Funding for the SDSS and SDSS-II has been provided by the Alfred P. Sloan Foundation, the Participating Institutions, the Nationa Science Foundation, the U.S. Department of Energy, the National Aeronautics and Space Administration, the Japanese Monbukagakusho, the Max Planck Society, and the Higher Education Funding Council for England. The SDSS Web Site is http://www.sdss.org/. The SDSS is managed by the Astrophysical Research Consortium for the Participating Institutions. The Participating Institutions are the American Museum of Natural History, Astrophysical Institute Potsdam, University of Basel, University of Cambridge, Case Western Reserve University, University of Chicago, Drexel University, Fermilab, the Institute for Advanced Study, the Japan Participation Group, Johns Hopkins University, the Joint Institute for Nuclear Astrophysics, the Kavli Institute for Particle Astrophysics and Cosmology, the Korean Scientist Group, the Chinese Academy of Sciences (LAMOST), Los Alamos National Laboratory, the Max-PlanckInstitute for Astronomy (MPIA), the Max-Planck-Institute for Astrophysics (MPA), New Mexico State University, Ohio State University, University of Pittsburgh, University of Portsmouth, Princeton University, the United States Naval Observatory, and the University of Washington. This research has made use of the NASA/IPAC Extragalactic Database (NED), which is operated by the Jet Propulsion Laboratory, California Institute of Technology, under contract with the National Aeronautics and Space Administration, and of NASA's Astrophysics Data System Bibliographic Services. We have extensively used and are grateful for the open source data analysis and visualisation tools: Matplotlib (Hunter 2007), SciPy (Jones et al. 2001-2015), hyper fit (Robotham \& Obreschkow 2015) and prettyplotlib (https://gi thub.com/ olgabot/prettyplotlib).

\section{References}

Abazajian, K. N., Adelman-McCarthy, J. K., Agüeros, M. A., et al. 2009, ApJS, 182,543

Adams, J. J., Gebhardt, K., Blanc, G. A., et al. 2012, ApJ, 745, 92

Aguerri, J. A. L., Méndez-Abreu, J., Falcón-Barroso, J., et al. 2015, A\&A, 576, A102

Amram, P., Marcelin, M., Balkowski, C., et al. 1994, A\&AS, 103, 5

Andersen, D. R., \& Bershady, M. A. 2003, ApJ, 599, L79

Balkowski, C., Bottinelli, L., Chamaraux, P., Gouguenheim, L., \& Heidmann, J. 1974, A\&A, 34, 43

Barrera-Ballesteros, J. K., Falcón-Barroso, J., García-Lorenzo, B., et al. 2014, A\&A, 568, A70

Bell, E. F., \& de Jong, R. S. 2001, ApJ, 550, 212

Bershady, M. A., Andersen, D. R., Harker, J., Ramsey, L. W., \& Verheijen, M. A. W. 2004, PASP, 116, 565

Bertola, F., Bettoni, D., Danziger, J., et al. 1991, ApJ, 373, 369

Bland, J., Taylor, K., \& Atherton, P. D. 1987, MNRAS, 228, 595

Blanton, M. R., Geha, M., \& West, A. A. 2008, ApJ, 682, 861

Böhm, A., \& Ziegler, B. L. 2007, ApJ, 668, 846
Böhm, A., Ziegler, B. L., Saglia, R. P., et al. 2004, A\&A, 420, 97 Bosma, A. 1978, Ph.D. Thesis, Groningen Univ., The Netherlands Bottinelli, L. 1971, A\&A, 10, 437

Calzetti, D. 2013, in Star Formation Rate Indicators, eds. J. Falcón-Barroso, \&

J. H. Knapen (Cambridge University Press), 419

Cappellari, M., \& Copin, Y. 2003, MNRAS, 342, 345

Cappellari, M., \& Emsellem, E. 2004, PASP, 116, 138

Catalán-Torrecilla, C., Gil de Paz, A., Castillo-Morales, A., et al. 2015, A\&A 584, A 87

Chan, T. K., Kereš, D., Oñorbe, J., et al. 2015, MNRAS, 454, 2981

Cole, S., Lacey, C. G., Baugh, C. M., \& Frenk, C. S. 2000, MNRAS, 319, 168

Courteau, S. 1996, ApJS, 103, 363

Courteau, S. 1997, AJ, 114, 2402

Courteau, S., \& Dutton, A. A. 2015, ApJ, 801, L20

Courteau, S., \& Rix, H.-W. 1999, ApJ, 513, 561

Courteau, S., Andersen, D. R., Bershady, M. A., MacArthur, L. A., \& Rix, H.-W. 2003, ApJ, 594, 208

Courteau, S., Dutton, A. A., van den Bosch, F. C., et al. 2007, ApJ, 671, 203

Cresci, G., Hicks, E. K. S., Genzel, R., et al. 2009, ApJ, 697, 115

Croton, D. J., Springel, V., White, S. D. M., et al. 2006, MNRAS, 365, 11

Davis, T. A., Bureau, M., Young, L. M., et al. 2011, MNRAS, 414, 968

Di Teodoro, E. M., Fraternali, F., \& Miller, S. H. 2016, A\&A, in press, DOI: $10.1051 / 0004-6361 / 201628315$

Dutton, A. A., \& van den Bosch, F. C. 2009, MNRAS, 396, 141

Dutton, A. A., van den Bosch, F. C., Dekel, A., \& Courteau, S. 2007, ApJ, 654 27

Dutton, A. A., Conroy, C., van den Bosch, F. C., et al. 2011, MNRAS, 416, 322

Eisenstein, D. J., \& Loeb, A. 1996, ApJ, 459, 432

Eke, V. R., Navarro, J. F., \& Steinmetz, M. 2001, ApJ, 554, 114

Emsellem, E., Cappellari, M., Krajnović, D., et al. 2007, MNRAS, 379, 401

Emsellem, E., Cappellari, M., Krajnović, D., et al. 2011, MNRAS, 414, 888

Falcón-Barroso, J., Lyubenova, M., \& van de Ven, G. 2015, in IAU Symp., 311, eds. M. Cappellari, \& S. Courteau, 78

Falcón-Barroso, J., Lyubenova, M., van de Ven, G., Mendez-Abreu, J., et al 2016, A\&A, in press, DOI: 10.1051/0004-6361/201628625

Flores, H., Hammer, F., Puech, M., Amram, P., \& Balkowski, C. 2006, A\&A, 455, 107

Foreman-Mackey, D., Conley, A., Meierjurgen Farr, W., et al. 2013, PASP, 125, 925

Franx, M., \& de Zeeuw, T. 1992, ApJ, 392, L47

García-Benito, R., Zibetti, S., Sánchez, S. F., et al. 2015, A\&A, 576, A135

García-Lorenzo, B., Márquez, I., Barrera-Ballesteros, J. K., et al. 2015, A\&A, 573, A59

Garrido, O., Marcelin, M., \& Amram, P. 2004, MNRAS, 349, 225

Giovanelli, R., Haynes, M. P., Herter, T., et al. 1997, AJ, 113, 53

Gnedin, O. Y., Weinberg, D. H., Pizagno, J., Prada, F., \& Rix, H.-W. 2007, ApJ, 671,1115

Gnerucci, A., Marconi, A., Cresci, G., et al. 2011, A\&A, 528, A88

Gonzalez, A. H., Williams, K. A., Bullock, J. S., Kolatt, T. S., \& Primack, J. R. 2000, ApJ, 528, 145

Goodman, J., \& Weare, J. 2010, Comm. Appl. Math. Comp. Sci., 5, 65

Governato, F., Willman, B., Mayer, L., et al. 2007, MNRAS, 374, 1479

Green, A. W., Glazebrook, K., McGregor, P. J., et al. 2014, MNRAS, 437, 1070

Hall, M., Courteau, S., Dutton, A. A., McDonald, M., \& Zhu, Y. 2012, MNRAS, 425, 2741

Hubble, E. P. 1926, ApJ, 64, 321

Hunter, J. D. 2007, Comp. Sci. Eng., 9, 90

Husemann, B., Jahnke, K., Sánchez, S. F., et al. 2013, A\&A, 549, A87

Jones, E., Oliphant, T., Peterson, P., et al. 2001-2015, SciPy: Open source scientific tools for Python [online; accessed 2015-06-17]

Kalinova, V., \& Lyubenova, M. 2016, Ph.D. Thesis, Univ. Heidelberg

Kalinova, V., van de Ven, G., Lyubenova, M., Falcón-Barroso, J., et al. 2016, MNRAS, submitted

Koda, J., Sofue, Y., \& Wada, K. 2000, ApJ, 532, 214

Krajnović, D., Bacon, R., Cappellari, M., et al. 2008, MNRAS, 390, 93

Maller, A. H., Berlind, A. A., Blanton, M. R., \& Hogg, D. W. 2009, ApJ, 691, 394

Marcelin, M., \& Athanassoula, E. 1982, A\&A, 105, 76

Masters, K. L., Springob, C. M., Haynes, M. P., \& Giovanelli, R. 2006, ApJ, 653, 861

McCarthy, I. G., Schaye, J., Font, A. S., et al. 2012, MNRAS, 427, 379

McGaugh, S. S., Schombert, J. M., Bothun, G. D., \& de Blok, W. J. G. 2000, ApJ, 533, L99

Miller, S. H., Bundy, K., Sullivan, M., Ellis, R. S., \& Treu, T. 2011, ApJ, 741, 115

Miller, S. H., Ellis, R. S., Sullivan, M., et al. 2012, ApJ, 753, 74

Miller, S. H., Sullivan, M., \& Ellis, R. S. 2013, ApJ, 762, L11

Mould, J. R., Huchra, J. P., Freedman, W. L., et al. 2000, ApJ, 529, 786 
Neistein, E., Maoz, D., Rix, H.-W., \& Tonry, J. L. 1999, AJ, 117, 2666 Neumayer, N., Walcher, C. J., Andersen, D., et al. 2011, MNRAS, 413, 1875 Nicholson, R. A., Bland-Hawthorn, J., \& Taylor, K. 1992, ApJ, 387, 503 Obreschkow, D., \& Meyer, M. 2013, ApJ, 777, 140

Opik, E. 1922, ApJ, 55, 406

Parzen, E. 1962, Ann. Math. Statist., 33, 1065

Persic, M., \& Salucci, P. 1991, ApJ, 368, 60

Persic, M., Salucci, P., \& Stel, F. 1996, MNRAS, 281, 27

Pizagno, J., Prada, F., Weinberg, D. H., et al. 2007, AJ, 134, 945

Puech, M., Flores, H., Hammer, F., et al. 2008, A\&A, 484, 173

Querejeta, M., Eliche-Moral, M. C., Tapia, T., et al. 2015, A\&A, 573, A78

Rix, H.-W., Guhathakurta, P., Colless, M., \& Ing, K. 1997, MNRAS, 285, 779

Roberts, M. S. 1969, AJ, 74, 859

Robotham, A. S. G., \& Obreschkow, D. 2015, PASA, 32, e033

Rodríguez, S., \& Padilla, N. D. 2013, MNRAS, 434, 2153

Rogstad, D. H., Wright, M. C. H., \& Lockhart, I. A. 1976, ApJ, 204, 703

Rosenblatt, M. 1956, Ann. Math. Statist., 27, 832

Roth, M. M., Kelz, A., Fechner, T., et al. 2005, PASP, 117, 620

Rubin, V. C., Burstein, D., Ford, Jr., W. K., \& Thonnard, N. 1985, ApJ, 289, 81

Saintonge, A., \& Spekkens, K. 2011, ApJ, 726, 77

Sánchez, S. F., Kennicutt, R. C., Gil de Paz, A., et al. 2012, A\&A, 538, A8

Sánchez, S. F., Rosales-Ortega, F. F., Iglesias-Páramo, J., et al. 2014, A\&A, 563 A49

Schmidt, M. 1968, ApJ, 151, 393

Schommer, R. A., Bothun, G. D., Williams, T. B., \& Mould, J. R. 1993, AJ, 105, 97

Scott, D. W., \& Sain, S. R. 2005, Handbook of Statistics, 24, 229

Shostak, G. S. 1975, ApJ, 198, 527

Silverman, B. W. 1986, Density estimation for statistics and data analysis, 26 (CRC press)
Steinmetz, M., \& Navarro, J. F. 1999, ApJ, 513, 555

Swinbank, A. M., Bower, R. G., Smith, G. P., et al. 2006, MNRAS, 368, 1631

Tonini, C., Maraston, C., Ziegler, B., et al. 2011, MNRAS, 415, 811

Trujillo-Gomez, S., Klypin, A., Primack, J., \& Romanowsky, A. J. 2011, ApJ, 742,16

Tully, R. B., \& Fisher, J. R. 1977, A\&A, 54, 661

Tully, R. B., \& Pierce, M. J. 2000, ApJ, 533, 744

Valdes, F., Gupta, R., Rose, J. A., Singh, H. P., \& Bell, D. J. 2004, ApJS, 152, 251

van den Bosch, F. C. 2000, ApJ, 530, 177

van den Bosch, F. C., Robertson, B. E., Dalcanton, J. J., \& de Blok, W. J. G. 2000, AJ, 119, 1579

Verheijen, M. A. W. 2001, ApJ, 563, 694

Verheijen, M. A. W., Bershady, M. A., Andersen, D. R., et al. 2004, Astron. Nachr., 325, 151

Vogelsberger, M., Genel, S., Sijacki, D., et al. 2013, MNRAS, 436, 3031

Vogelsberger, M., Genel, S., Springel, V., et al. 2014, Nature, 509, 177

Vogt, N. P., Forbes, D. A., Phillips, A. C., et al. 1996, ApJ, 465, L15

Walcher, C. J., Lamareille, F., Vergani, D., et al. 2008, A\&A, 491, 713

Walcher, C. J., Wisotzki, L., Bekeraité, S., et al. 2014, A\&A, 569, A1

Weijmans, A.-M., Krajnović, D., van de Ven, G., et al. 2008, MNRAS, 383, 1343

Wild, V., Charlot, S., Brinchmann, J., et al. 2011a, MNRAS, 417, 1760

Wild, V., Groves, B., Heckman, T., et al. 2011b, MNRAS, 410, 1593

Williams, M. J., Bureau, M., \& Cappellari, M. 2009, in Galaxy Evolution: Emerging Insights and Future Challenges, eds. S. Jogee, I. Marinova, L. Hao, \& G. A. Blanc, ASP Conf. Ser., 419, 167

Yegorova, I. A., \& Salucci, P. 2007, MNRAS, 377, 507

Ziegler, B. L., Böhm, A., Fricke, K. J., et al. 2002, ApJ, 564, L69 